\title{
Robot-based Indoor Positioning of UHF-RFID Tags: the SAR Method with Multiple Trajectories
}

\author{
Fabio Bernardini, Student Member, Alice Buffi, Member, IEEE, Daniele Fontanelli, Senior Member, \\ IEEE, David Macii, Senior Member, IEEE, Valerio Magnago, Mirko Marracci, Member, IEEE, \\ Andrea Motroni, Student Member, Paolo Nepa, Senior Member, IEEE, Bernardo Tellini, Senior \\ Member, IEEE
}

\begin{abstract}
This paper presents the application of the Synthetic Aperture Radar (SAR) localization method for indoor positioning of UHF-RFID tags when the robot-mounted reader antenna moves alongside multiple trajectories. By properly combining the phase data associated to a set of multiple paths, the synthetic apertures along the main directions enlarge and then the localization accuracy may improve. Besides, during consecutive inventory rounds, several tag position estimates are available and they can be profitably combined to minimize the localization uncertainty. Different combination approaches are investigated to determine the best choice to improve the localization performance. The method capabilities are discussed through a numerical analysis, by considering different configurations of the multiple apertures and different sources of measurement uncertainty. Finally, the proposed localization method is validated through an experimental analysis carried out with commercial RFID hardware and a robotic wheeled walker, in an indoor scenario, by employing different types of tags. The knowledge of the reader/robot trajectory required by the SAR method is here achieved with an optical system.
\end{abstract}

Index Terms - Moving RFID reader; RFID robot; Robot-based Localization; Synthetic Aperture Radar localization; Tag indoor localization; UHF-RFID position measurement, UHF-RFID.

\section{INTRODUCTION}

$\mathrm{I}$ $\mathrm{N}$ the last years, indoor positioning systems received great attention in several applications such as item localization in smart warehouses, worker/tool localization in smart factories, and people localization in large environments, e.g. hospitals, retirement homes, airports, and shopping malls. When positioning systems are considered, several issues have to be addressed: infrastructure complexity and cost, system scalability and robustness, operational life of devices, achievable accuracy and precision [1]. A large spectrum of technologies exist, which are based on vision-based systems [2], laser range finders [3], sonars [4], Wi-Fi [5], Bluetooth [6], Ultra-Wide Band [7], magnetic fields [8], encoders and/or

Manuscript received July 3, 2020.

This work was supported in part by Regione Toscana (POR FESR 20142020 - Line 1 - R\&D Strategic Projects) under project IREAD4.0 (CUP number 7165.24052017.112000028). This work was supported in part by the Italian Ministry of Education and Research (MIUR) in the framework of the CrossLab project (Departments of Excellence) (Corresponding author: Alice Buffi).

A. Buffi, M. Marracci and B. Tellini are with the Department of Energy, Systems, Territory and Constructions Engineering, University of Pisa, Pisa, Italy (e-mail: alice.buffi@unipi.it,_mirko.marracci@unipi.it, bernardo.tellini@unipi.it). inertial sensors [9], and Radio Frequency Identification (RFID) [10]. A survey of the main features of each of the abovementioned technologies can be found in [1].

In warehouses [11], retail scenarios [12] and libraries [13], a large number of items has to be managed; thus, passive UHFRFID technology represents a promising solution by virtue of its low cost and easiness of installation. It offers tangible benefits to both suppliers and retailers, maximizing consumer value and minimizing supply-chain inefficiencies [14]-[15]. Unlike the barcode technology, it does not require line-of-sight (LOS) condition and a precise alignment between reader antenna and tagged items. Moreover, unlike RFID technologies at lower frequencies, it guarantees a reading range of several meters, and the wide-beam reader antennas along with the anticollision protocol implemented in the EPC Global C1 G2 standard can provide connectivity with multiple tags at the same time.

Real-time inventory can be performed in several ways: with portable readers [16], through fixed readers installed on the ceiling [17], via mobile robot equipped with UHF-RFID reader [18]-[19], or with ad-hoc smart shelf integrating UHF-RFID reader antennas [20]-[21]. Besides the tag identification, the RFID technology can be used also for tag sorting [22]-[23], or tag localization [18].

Among commercial solutions, some RFID manufacturers have developed systems employing phased-array antennas to create a narrow and steerable beam, by enabling simultaneous tag identification and localization [24]. One of them is the Impinj xArray [25] system able to detect and locate tags in large-scale RFID scenarios (e.g. retail, healthcare and manufacturing), with an average positioning error of about 1.5 meters. Other commercial solutions rely on Unmanned Ground Vehicles (UGV) equipped with a UHF-RFID reader able to self-navigate inside the environment, as well as to provide the antenna motion and to acquire data from RFID tags in order to make the inventory, while possibly finding the position of different items. In particular, AdvanRobot by Keonn [26],

D. Fontanelli and D. Macii are with the Department of Industrial Engineering, University of Trento, Trento, Italy (e-mail: daniele.fontanelli@unitn.it, david.macii@unitn.it).

V. Magnago is with the Department of Information Engineering and Computer Science, University of Trento, Trento, Italy (e-mail: valerio.magnago@unitn.it).

F. Bernardini, A. Motroni and P. Nepa are with the Department of Information Engineering, University of Pisa, Pisa, Italy (e-mail: andrea.motroni@ing.unipi.it, paolo.nepa@unipi.it). 
already employed in Walmart warehouses, Tory by MetraLabs [27], and Robi by Fetch Robotics [28] are worth mentioning.

In this paper, the SAR-based localization method [29] is applied when the robot-mounted RFID reader antenna moves along multiple trajectories. The proper combination of the phase data collected over different independent trajectories is investigated for localization accuracy improvement. Besides, when consecutive tag position estimates are available due to consecutive robot inventory rounds, the criteria to determine the solution that minimizes the localization uncertainty is discussed.

The paper is organized as follows. After the related work description in Section II, the SAR-based localization method exploiting multiple trajectories is presented in Section III. Then, the numerical analysis is described in Section IV, by comparing the performance of the SAR method with several multipletrajectory cases. Indeed, the experimental setup with a reader antenna carried out by a robotic wheeled walker is described in Section V, together with the localization performance for multiple tags of different types, in a real indoor environment. Finally, conclusions and future research directions are drawn in Section VI.

\section{RELATED WORKS}

Generally speaking, when realizing a UHF RFID-based positioning system, classic solutions exploit the amplitude of the signal backscattered by the targeted tag, which is measured through the Received Signal Strength Indicator (RSSI). Such a parameter is strongly dependent on the environment and it suffers from multipath propagation, tag type, tag orientation, tagged item material and so on. Consequently, the definition of a reliable path loss model is not an easy task, especially in indoor scenarios, and the expected localization uncertainty could be so large as some meters. As an alternative, sceneanalysis approaches can be adopted. In [12], the authors presented a Bayesian filter-based algorithm, called BFVP (Bayesian filter of variable RF transmission power). In an offline phase, the robot navigates inside the scenario to fully cover the target space and collect observations of RFID tags at variable RF power. The constructed detection profiles are then used during the online phase to locate the tags. This method exhibits a localization uncertainty of about $50 \mathrm{~cm}$ in a retail environment with strong multipath phenomena. To improve localization accuracy, the reference tags are typically employed with proper modifications of classical $k$-Nearest Neighbour $(k$ $\mathrm{NN}$ ) algorithms [29]-[31]. In [32] the authors propose a system with a moving reader antenna measuring the multipath profiles of the reference tags to enhance tag localization accuracy, according to the insight that nearby tags have similar multipath effects. The use of reference tags was also combined with machine-learning techniques such as the Support Vector Regression [33], Artificial Neural Networks [34] and the Naïve Bayes algorithm [35].

To achieve a better localization accuracy, the phase-based methods can be employed [36]. They typically exploit the Phase Difference of Arrival (PDOA) of the tag backscattered signal, to mitigate the effects of the propagation channel. The Time Domain PDOA (TD-PDOA) method allows to estimate the relative radial speed between tags and antennas through phase measurements at different instants. This solution is robust in rich multipath scenarios, but a fine tag localization cannot be performed. The Frequency Domain PDOA (FD-PDO) method instead exploits the phase measurements at two or more frequencies to estimate the tag distance. It can be applied for both moving and stationary tags being similar to the Frequency Modulation (FM) continuous-wave (CW) radar [37]. However, such a technique suffers from the quite limited bandwidth of RFID systems, especially in Europe (ETSI band 865-868 $\mathrm{MHz}$ ). With the Spatial Domain PDOA (SD-PDOA) method, the Direction-of-Arrival (DoA) of the tag signal can be measured by employing two or more antennas. As a result, the tag position can be determined from multiple distance/angle estimates, by applying multilateration or triangulation [38].

Among the SD-PDOA techniques, methods exist which are based on the Synthetic Aperture Radar (SAR) approach with multiple reader antennas [39], or with a moving reader antenna creating a virtual array [40]. Such a method exploits the relative motion of the reader antenna with respect to the tagged items, to locate them. The antenna can be moved through a handling system [41], a robotic arm [42], an Unmanned Ground Vehicle (UGV) [43]-[44], or an Unmanned Aerial Vehicle (UAV) [45]. The main requirement for the application of this method is the knowledge of the reader antenna trajectory. The lower the measurement uncertainty of a given trajectory is, the smaller the tag position measurement uncertainty becomes. The SARapproach is also employed in [46] for tag localization based on a multiple-input multiple-output system and ad-hoc hardware. In [47], the concept of multiple baseline synthetic array is applied to conventional SAR-based methods, to elaborate the phase samples collected progressively along the reader trajectory. In [48], the authors present a 3D localization system to estimate the height of tagged items, by exploiting an Interferometric SAR approach. To solve the phase ambiguity, a density-based spatial clustering method is applied. The method median error of this method is $24 \mathrm{~cm}$, in an indoor office environment with six targeted tags. In [49], the authors propose a phase unwrapping approach together with a Non-Linear Optimization algorithm, named as Phase ReLock. The robot moves over a planar trajectory measured through a laser-based system, on a plane parallel to the plane the tag lies on. The experimental analysis is carried out by employing 80 tags in an area of $10 \mathrm{~m} \times 2.5 \mathrm{~m}$. 2D localization performance is comparable with the one achievable with conventional SARbased method, which is regarded as the accuracy benchmark in the literature. This method has been extended to 3D localization as well [50], by employing a non-straight robot path. In [44], the authors present a smart system called RF-Scanner, which is able to locate items placed in bookshelves and to discover lying-down books. A phase-based method based on a fitting hyperbola model can ensure a centimetre level uncertainty.

To improve localization accuracy, multiple antennas can also be mounted on the robot [51]-[53]. In [52], two reader antennas aligned along the vertical direction are installed on the robot. The SAR-based method is applied separately to the phase samples collected by each antenna and the obtained matching function are then combined to get a $3 \mathrm{D}$ localization with a centimetre order error. 


\section{The MultiPle-APERTURe SAR-BASED Methodology}

Fig. 1 depicts a typical indoor warehouse with a robot equipped with RFID hardware, and several items tagged with passive transponders (tags). In UHF-RFID systems, the reader transmitted power and the antenna gain are limited by international regulations. Thus, the detection range mainly depends on the tag chip sensitivity. In last-generation tag chips, passive tags can be detected at distance larger than $10 \mathrm{~m}$, allowing the passive UHF-RFID technology to implement an effective real-time inventory in indoor scenarios. A wide-beam reader antenna is installed on the robot to detect many tags which can be distinguished through their own Electronic Product Code (EPC).

The robot movement with respect to the static tags allows to improve the inventory performance by mitigating the adverse effects of the shadowing and multipath phenomena. Besides, such reader-tag relative motion allows to apply the SAR-based localization method proposed by the authors in [40] to estimate the position of every detected tag. The main requirement is the knowledge of the reader antenna trajectory, which can be measured through a vision-based system in an indoor scenario, as described in this paper.

During robot inventory, the reader antenna detects each tag along one or more portions of its whole path, depending on several factors: the trajectory shape, the relative orientation between the antenna and the tag, the reader antenna Half Power Beam Width (HPBW), the obstacles in the indoor scenario. In this case, the SAR-based method can be applied to the phase samples acquired during multiple apertures provided that the sampling theorem requirement is satisfied [56]. Besides, during consecutive inventory rounds, several tag position estimates are available and this can be profitably used to improve the localization performance.

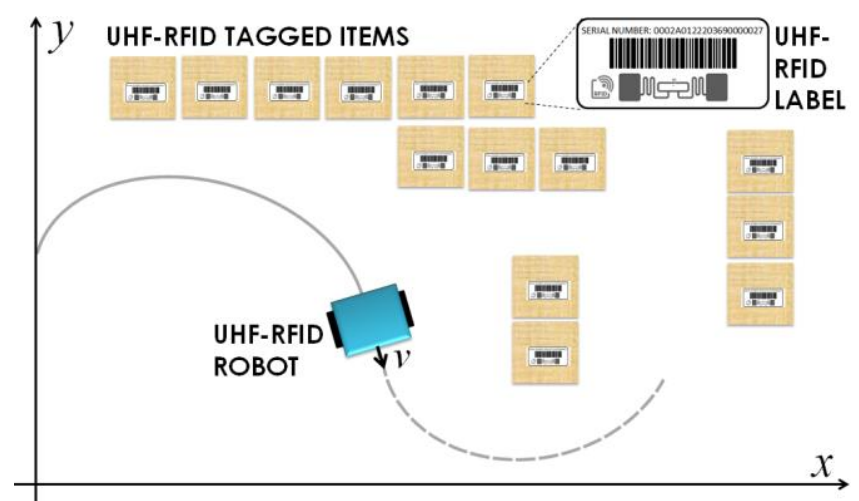

Fig. 1. Sketch of tagged items in an indoor warehouse, where a robot equipped with a UHF-RFID hardware can identify and localize each detected tag.

\section{A. The Multiple Aperture SAR-based Localization Method}

When the reader antenna on the robot moves among tags, the phase of the backscattered complex signal (briefly called as complex signal hereafter) depends on the relative distance $r_{n}$ between the reader antenna and the tag position $\mathbf{p}_{\text {tag }}=$ $\left[x_{t a g}, y_{t a g}, z_{t a g}\right]$ as:

$$
\phi_{n}\left(\mathbf{p}_{\text {tag }}\right)=4 \pi r_{n} / \lambda+\phi_{0}, \quad n=1, \ldots, N_{r}
$$

where $\lambda$ is the free-space wavelength of the radiated field, $N_{r}$ is the number of available successful readings while $\phi_{0}$ is the phase offset including the effect of cables and other reader components [36]. In particular, in our model $\phi_{0}$ is assumed to be constant within the reader antenna main beam. Thus, the phase variations along the robot trajectory are related to the variations of the distance $r_{n}$ :

$$
r_{n}=\left\|\mathbf{p}_{\mathbf{n}}-\mathbf{p}_{\text {tag }}\right\|, \quad n=1, \ldots, N_{r}
$$

In (2), $\|\cdot\|$ denotes the $\mathrm{L} 2$ norm of the distance vector between the unknown tag position $\mathbf{p}_{\text {tag }}$ and the reader antenna position $\mathbf{p}_{\mathbf{n}}=\left[x_{n}, y_{n}, z_{n}\right]$ of the $n$-th reading along the robot path. The latter can be modelled as:

$$
\mathbf{p}_{\mathbf{n}}=\mathbf{p}_{\mathrm{Rn}}+\Delta \mathbf{p}_{\mathrm{R}-\mathrm{APCn}}
$$

where, $\mathbf{p}_{\mathbf{R n}}=\left[x_{R n}, y_{R n}, z_{R n}\right]$ is the instantaneous position of the robot rotation centre and $\Delta \mathbf{p}_{\mathbf{R}-\mathbf{A P C n}}$ is the relative position of the RFID antenna phase centre with respect to the robot barycentre at the time of the $n$-th reading.

We consider the variations of the complex signal phase with respect to the value assumed at an assigned reference time, namely the relative phase history of the complex signal. Therefore, the constant $\phi_{0}$ is canceled. Let us assume the first available reading as our reference. Thus, from (1) it follows that:

$$
\Delta \phi_{n}=\phi_{n}-\phi_{1}, \quad n=1, \ldots, N_{r}
$$

The resulting normalized phasor sequence can be written as:

$$
\mathbf{y}\left(\mathbf{p}_{\text {tag }}\right)=\left[\begin{array}{ll}
1, & e^{-j\left(\phi_{2}-\phi_{1}\right)}, \ldots, e^{-j\left(\phi_{N_{r}}-\phi_{1}\right)}
\end{array}\right]^{T}
$$

being ' $T$ ' the transpose operator. For a given path of the reader antenna, $\mathbf{y}\left(\mathbf{p}_{\text {tag }}\right)$ varies as a function of the actual tag position.

As already said, when the robot moves within the indoor scenario, the reader antenna can collect data from the tag during several trajectories, even not contiguous in time. The collected phase samples (1) can be jointly employed to build the normalized phasor sequence (5), provided that, the average spatial sampling satisfies the sampling theorem [56], namely the condition:

$$
\Delta=\frac{1}{N_{r}-1} \sum_{n=1}^{N_{r}-1}\left\|\mathbf{p}_{\mathbf{n}+\mathbf{1}}-\mathbf{p}_{\mathbf{n}}\right\| \leq \lambda /[4 \sin (H P B W / 2)]
$$

Above procedure works well as a result of the adopted phasor sequence, which allows to tackle the phase ambiguity problem.

The lengths of the synthetic apertures along the three main directions can be defined as: 


$$
\left\{\begin{array}{l}
D_{x}=\max _{n=1, \ldots, N_{r}}\left\{x_{n}\right\}-\min _{n=1, \ldots, N_{r}}\left\{x_{n}\right\} \\
D_{y}=\max _{n=1, \ldots, N_{r}}\left\{y_{n}\right\}-\min _{n=1, \ldots, N_{r}}\left\{y_{n}\right\} \\
D_{z}=\max _{n=1, \ldots, N_{r}}\left\{z_{n}\right\}-\min _{n=1, \ldots, N_{r}}\left\{z_{n}\right\}
\end{array}\right.
$$

While the total length of the synthetic aperture is:

$$
D_{t o t}=\sum_{n=1}^{N_{r}-1}\left\|\mathbf{p}_{\mathbf{n}+\mathbf{1}}-\mathbf{p}_{\mathbf{n}}\right\|
$$

For a defined robot trajectory, composed by multiple notcontiguous apertures, a set of nominal normalized phasor sequences can be computed analytically, for hypothesized values $\mathbf{p}_{\text {tag }}^{\prime}=\left[x_{\text {tag }}^{\prime}, y_{\text {tag }}^{\prime}, z_{\text {tag }}^{\prime}\right]$ of the tag spatial coordinate $\mathbf{p}_{\text {tag }}$, based on (5), i.e.

$$
\mathbf{a}\left(\mathbf{p}_{\text {tag }}^{\prime}\right)=\left[1, e^{-j\left(\phi_{2}^{\prime}-\phi_{1}^{\prime}\right)}, \ldots, e^{-j\left(\phi_{N_{r}}^{\prime}-\phi_{1}^{\prime}\right)}\right]^{T}
$$

where $\phi_{n}^{\prime}=\phi_{n}\left(\mathbf{p}_{\text {tag }}^{\prime}\right)$ is given by (1). The $\mathbf{p}_{\text {tag }}^{\prime}$ values belong to a grid of points whose step corresponds to the method resolution.

The best match between (5) and (9) can be found through the normalized spatial-domain cross-correlation (matching function):

$$
\mathbf{C}\left(\mathbf{p}_{\text {tag }}^{\prime}\right)=\frac{\left|\mathbf{a}^{\mathrm{H}}\left(\mathbf{p}_{\text {tag }}^{\prime}\right) \mathbf{y}\left(\mathbf{p}_{\text {tag }}\right)\right|}{\left\|\mathbf{a}^{\mathrm{H}}\left(\mathbf{p}_{\text {tag }}^{\prime}\right)\right\| \cdot\left\|\mathbf{y}\left(\mathbf{p}_{\text {tag }}\right)\right\|}
$$

with ' $\mathrm{H}$ ' being the Hermitian operator. The cross correlation in (10) is calculated over the above-mentioned grid of $\mathbf{p}_{\text {tag }}^{\prime}$ values. As already stated in [45], the position of the primary peak $C_{\max }$ of (10), represents the most likely estimate of the tag spatial coordinates $\widehat{\mathbf{p}}_{\text {tag }}=\left[\hat{x}_{\text {tag }}, \hat{y}_{\text {tag }}, \hat{z}_{\text {tag }}\right]$ :

$$
\widehat{\mathbf{p}}_{\text {tag }}=\underset{\mathbf{p}_{\text {tag }}^{\prime}}{\arg \max } \mathbf{C}\left(\mathbf{p}_{\text {tag }}^{\prime}\right)
$$

The ratio between the peak value $C_{\max }$ and the secondary peak value $C_{s p}$ is defined as Peak-to-Secondary-Peak-Level (PSPL):

$$
\boldsymbol{P S P L}=\frac{C_{\max }}{C_{s p}}
$$

The higher the peak value $C_{\text {max }}$, the greater the similarity between the tag phasor sequence and the nominal one. Moreover, the presence of a secondary peak $\mathbf{p}_{\mathbf{s p}}=$ $\left[x_{s p}, y_{s p}, z_{s p}\right]$ with a low associated value $C_{s p}$ reduces the uncertainty in the primary peak determination.

\section{B. Consecutive estimations}

Several tag position estimates can be computed over time by processing phase data acquired during different set of multiple trajectories. This can be fruitfully employed to improve the localization performance. If $\widehat{\mathbf{p}}_{\text {tag }}(\boldsymbol{k})$ for $k \in\left\{1, \ldots, N_{e}\right\}\left(N_{e}\right.$ being the number of available estimates) is the $k$-th available estimate obtained with a peak value of $C_{\max }(k)$ and a Peak-toSecondary-Peak-Level of $P S P L(k)$, different criteria can be adopted.

The first one selects the solution with the highest value of the $C_{\max }(k)$ parameter:

$$
\widehat{\mathbf{p}}_{\text {tag-best1 }}=\left.\widehat{\mathbf{p}}_{\text {tag }}\right|_{\max \left\{C_{\max }(k)\right\}}
$$

with $k \in\left\{1, \ldots, N_{e}\right\}$.

The second one aims at selecting the solution with the highest value of the $P S P L(k)$ parameter:

$$
\widehat{\mathbf{p}}_{\text {tag-best2 }}=\left.\widehat{\mathbf{p}}_{\text {tag }}\right|_{\max \{P S P L(k)\}}
$$

The third approach is based on an average operation among consecutive position estimates:

$$
\widehat{\mathbf{p}}_{\text {tag-avg }}=\frac{\mathbf{1}}{\boldsymbol{N}_{\boldsymbol{e}}} \sum_{k=1}^{N_{e}} \widehat{\mathbf{p}}_{\text {tag }}(\boldsymbol{k})
$$

We further consider the possibility to process the phase data collected by a tag during consecutive multiple trajectories altogether, provided that the spatial sampling condition (6) is met. Thus, this last criterion is also considered in the following numerical analysis to evaluate the best approach minimizing the localization uncertainty.

\section{Characterization of the Measurement Method WITH MULTIPLE TRAJECTORIES}

\section{A. Multiple trajectories}

A numerical analysis was carried out to characterize the SAR measurement method with multiple trajectories. We consider an RFID reader antenna moving along a planar (2D) trajectory, parallel to the $x y$ plane, as illustrated in Fig. 1. We firstly investigate four different shapes of the 2D trajectories followed by the reader, as represented in Fig. 2: an L-shape path from point $A=[0,0,0.5] \mathrm{m}$, to point $B=[2,0,0.5] \mathrm{m}$, up to point $C=[2,2,0.5] \mathrm{m}$ (Fig. 2a); a C-shape trajectory, from point $A$, to point $B$, to point $C$, up to point $D=[0,2,0.5] \mathrm{m}$ (Fig. 2b); a double straight-line trajectory composed by two paths, the first one from point $A$, to point $B$ and the second one from point $C$ to point $D$ (Fig. 2b); a Cross-shape trajectory composed by two paths, the first from point $A$, to point $B$ and the second one from point $E=[0,-1,0.5] \mathrm{m}$, to point $F=$ $[0,1,0.5] \mathrm{m}$ (Fig. 2a). The considered trajectories have the same synthetic apertures along the $x$-direction $\left(D_{x}=2 \mathrm{~m}\right)$ and the $y$-direction $\left(D_{y}=2 \mathrm{~m}\right)$. However, they have a different total length: $D_{t o t-L}=4 \mathrm{~m}, D_{t o t-C}=D_{t o t-D R}=6 \mathrm{~m}$ and $D_{\text {tot-CROSS }}=5.41 \mathrm{~m}$. The average spatial sampling for the three trajectories are: $\Delta_{L}=0.02 \mathrm{~m}, \Delta_{C}=0.02 \mathrm{~m}, \Delta_{D R}=$ $0.03 \mathrm{~m}$, and $\Delta_{\text {CROSS }}=0.027 \mathrm{~m}$, for the L-shape, the C-shape, the double straight lines and the Cross-shape trajectories, respectively. In all cases, the sampling theorem is satisfied [56]. 


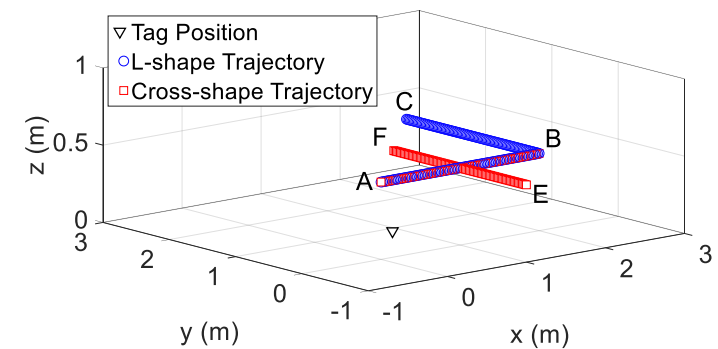

(a)

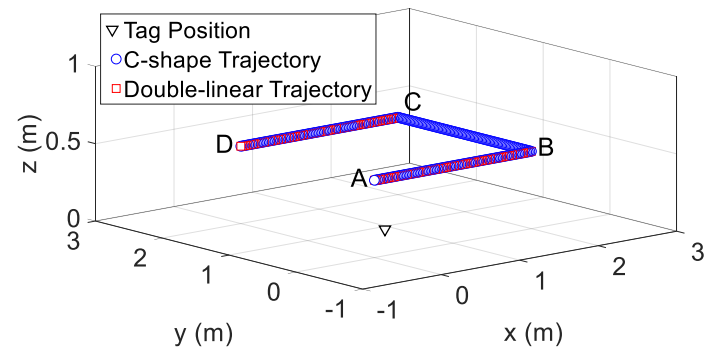

(b)

Fig. 2. The different types of trajectories considered in the simulations: (a) L-shape (circle markers) and Cross-shape (square markers) and (b) C-shape (circle markers) and double straight-line (square markers).

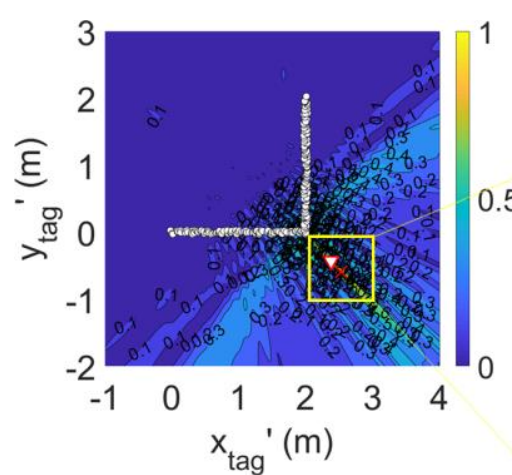

(a)

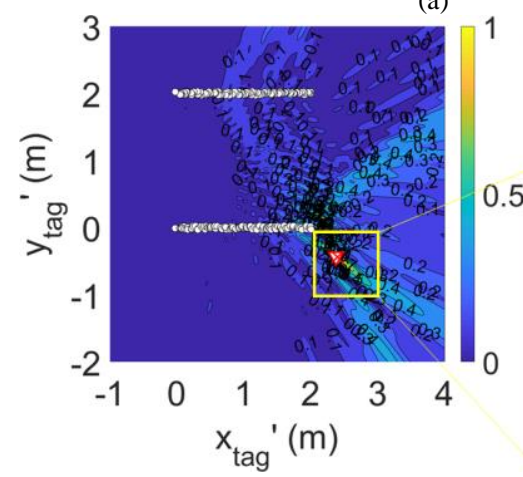

(c)
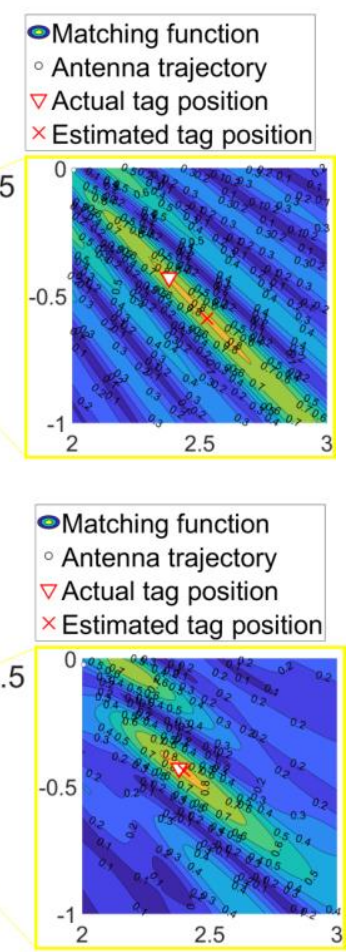

Fig. 3. Matching function and the corresponding estimated tag position by applying the SAR-based method for the different types of trajectories: (a) L-shape,

(b) C-shape, (c) double straight-line, and (d) Cross-shape.

In the numerical analysis, we assume a uniformly distributed error in the interval between $-3 \mathrm{~cm}$ and $3 \mathrm{~cm}$ for the reader antenna positions. The operating frequency is set at $f_{0}=$ 865.7 MHz. Then, the standard deviation of phase measurements is chosen equal to $\sigma_{\phi}=0.1 \mathrm{rad}$ [54]. Through the numerical analysis of this section, the considered target region is a $6 \mathrm{~m} \times 6 \mathrm{~m}$ grid with a step of $1 \mathrm{~cm}$ centred in $[1,1,0] \mathrm{m}$. Without loss of generality, we suppose to know the tag $z$-coordinate $\left(z_{\text {tag }}\right)$ and the analysis is carried out in terms of $2 \mathrm{D}$ localization performance. For the more general case of a fully 3D localization of items placed at arbitrary $z$-coordinates, the proposed localization method can still be applied by varying the hypothesized values of all three coordinates $\left[x_{t a g}^{\prime}, y_{t a g}^{\prime}, z_{t a g}^{\prime}\right]$, instead of two only $\left[x_{t a g}^{\prime}, y_{t a g}^{\prime}\right]$. Suitable optimization techniques such as the particle swarm optimization can be employed to reduce the computational burden [52], with respect to a standard full-space search method. Fig. 3 shows the matching function on the $z=0$ plane by applying the SAR-based localization method for the trajectories of Fig. 2, when the targeted tag is placed at $\mathbf{p}_{\mathrm{tag}}=$
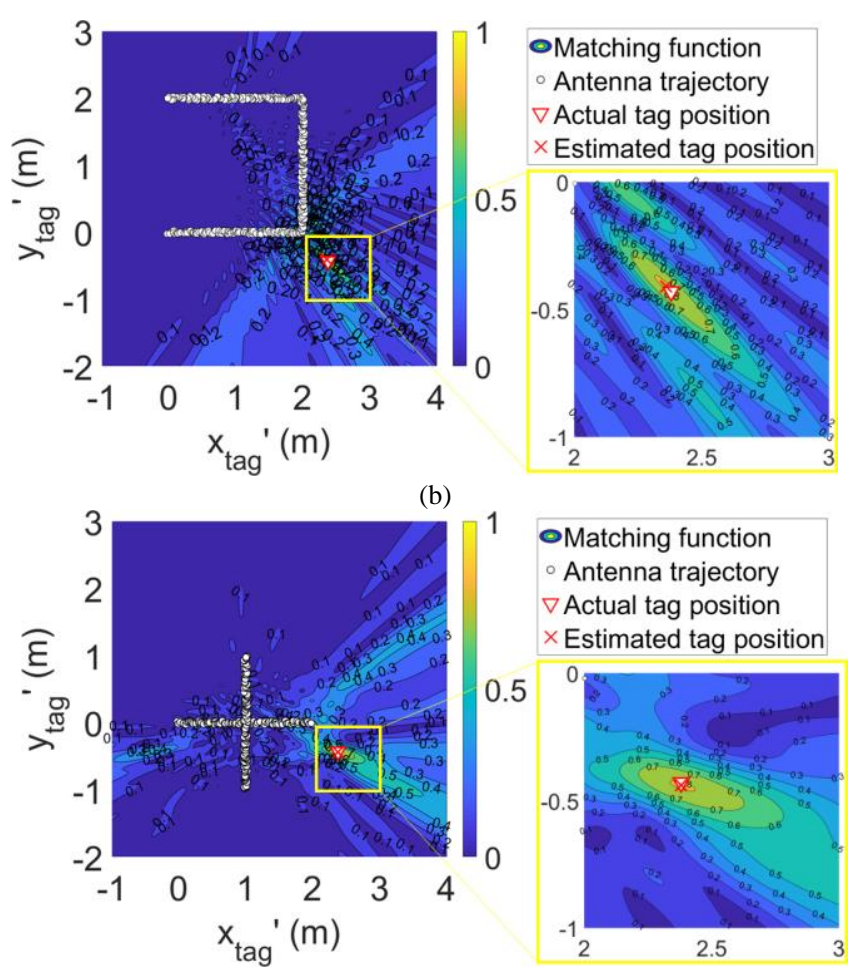

$[2.38,-0.43,0.00] \mathrm{m}$. For the first three trajectories the main lobe sizes (Table I) are quite similar, while they are slightly different for the Cross-shape trajectory. This confirms that the matching function shape depends on several factors such as the synthetic aperture lengths along different directions and the antenna-tag distance [55]. The corresponding primary and secondary peak values are reported in Table I along with the $3 \mathrm{~dB}$ sizes $\left(W_{x}\right.$ and $\left.W_{y}\right)$ of the main lobe along the axes of the chosen reference frame. The L-shape and the C-shape trajectories ensure a clear peak detection, with a low value of the secondary peak. Indeed, the double straight-line trajectory exhibits a high secondary peak, which however does not influence the primary peak significantly and it shows similar localization performance of the $\mathrm{C}$-shape trajectory due to the fact that they have the same total synthetic aperture $\left(D_{\text {tot }}\right)$. Indeed, the Cross-shape trajectory provides the higher PSPL value and, consequently, ensures a lower localization uncertainty. To better understand the influence of a high secondary peak which typically appears when multiple trajectories are considered, some Monte Carlo simulations are 
carried out for different tag positions. Indeed, the shape of the matching function is strictly related to the reader antenna trajectory with respect to the tag position itself. In details, 10000 tag positions are considered as randomly distributed over the $z=0$ plane, in a $6 \mathrm{~m} \times 6 \mathrm{~m}$ area centred in $[1,1,0] \mathrm{m}$, with $z_{\text {tag }}=0 \mathrm{~m}$. Firstly, we investigate the number of cases in which the matching function exhibits a high secondary peak, namely when the secondary peak is higher than $C_{\text {max }} / \sqrt{2}$. The results are shown in Fig. 4 as a function of the synthetic aperture length along the $y$-direction, $D_{y}$, for the L-shape (circle blue markers), the C-shape (square red markers), the double straightline (triangle green markers) and the Cross-shape path (star black markers) trajectories.

TABle I. Parameters of the Matching Function in Fig. 3 By APPlying THE SAR-BASED LOCALIZATION METHOD FOR DIFFERENT TRAJECTORIES.

\begin{tabular}{|l|l|l|l|l|}
\hline Trajectory & L-shape & C-shape & $\begin{array}{l}\text { Double } \\
\text { straight-line }\end{array}$ & Cross-shape \\
\hline$D_{x}$ & $2 \mathrm{~m}$ & $2 \mathrm{~m}$ & $2 \mathrm{~m}$ & $2 \mathrm{~m}$ \\
\hline$D_{y}$ & $2 \mathrm{~m}$ & $2 \mathrm{~m}$ & $2 \mathrm{~m}$ & $2 \mathrm{~m}$ \\
\hline$D_{\text {tot }}$ & $4 \mathrm{~m}$ & $6 \mathrm{~m}$ & $6 \mathrm{~m}$ & $5.41 \mathrm{~m}$ \\
\hline$\Delta$ & $2 \mathrm{~cm}$ & $2 \mathrm{~cm}$ & $3 \mathrm{~cm}$ & $2.7 \mathrm{~cm}$ \\
\hline$C_{\max }$ & 0.81 & 0.81 & 0.83 & 0.81 \\
\hline$C_{s p}$ & 0.68 & 0.67 & 0.79 & 0.55 \\
\hline$P S P L$ & 1.19 & 1.21 & 1.05 & 1.47 \\
\hline$W_{x}$ & $16.0 \mathrm{~cm}$ & $15.5 \mathrm{~cm}$ & $18.0 \mathrm{~cm}$ & $47.0 \mathrm{~cm}$ \\
\hline$W_{y}$ & $16.0 \mathrm{~cm}$ & $15.5 \mathrm{~cm}$ & $15.0 \mathrm{~cm}$ & $19.0 \mathrm{~cm}$ \\
\hline$\hat{x}_{\text {tag }}$ & $2.53 \mathrm{~m}$ & $2.36 \mathrm{~m}$ & $2.40 \mathrm{~m}$ & 2.38 \\
\hline$\hat{y}_{t a g}$ & $-0.59 \mathrm{~m}$ & $-0.41 \mathrm{~m}$ & $-0.44 \mathrm{~m}$ & -0.43 \\
\hline$x_{s p}$ & $2.16 \mathrm{~m}$ & $2.20 \mathrm{~m}$ & $2.18 \mathrm{~m}$ & 2.96 \\
\hline$y_{s p}$ & $0.00 \mathrm{~m}$ & $-0.04 \mathrm{~m}$ & $-0.04 \mathrm{~m}$ & -0.80 \\
\hline
\end{tabular}

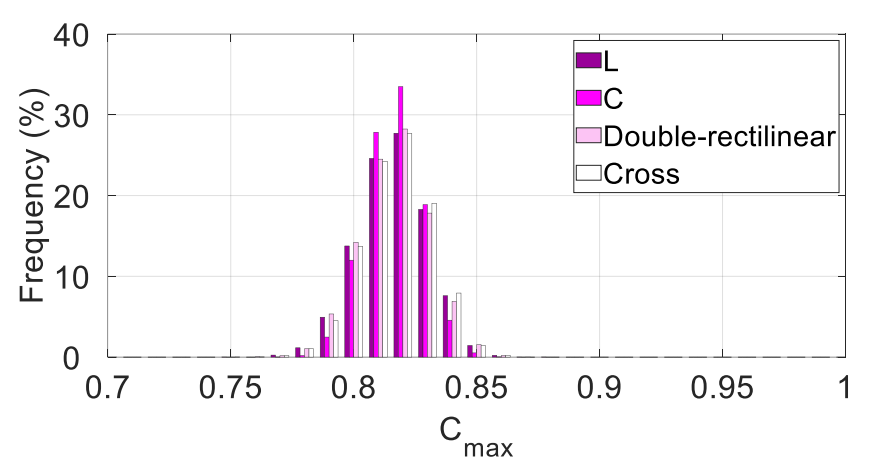

(a)
As the $D_{y}$ value increases, the number of appearances of the secondary peaks decreases for all the considered trajectories, except for the double straight-line one. This is due to the fact that even if the total synthetic aperture increases, the distance between the two trajectory portions increases as well.

The histograms of the primary peak $C_{\max }$ (Fig. 5a) and the Peak-to-Secondary-Peak-Level PSPL (Fig. 5b) are represented in Fig. 5 by considering the three trajectories for the case of $D_{y}=2 \mathrm{~m}$, namely when they have the same synthetic apertures along both the $x$ - and the $y$-directions. The histograms of the positioning errors $\epsilon_{x}=\hat{x}_{\text {tag }}-x_{\text {tag }}$ and $\epsilon_{y}=\hat{y}_{\text {tag }}-y_{\text {tag }}$ a along the $x$-and the $y$-axis, respectively, are depicted in Fig. 6 .

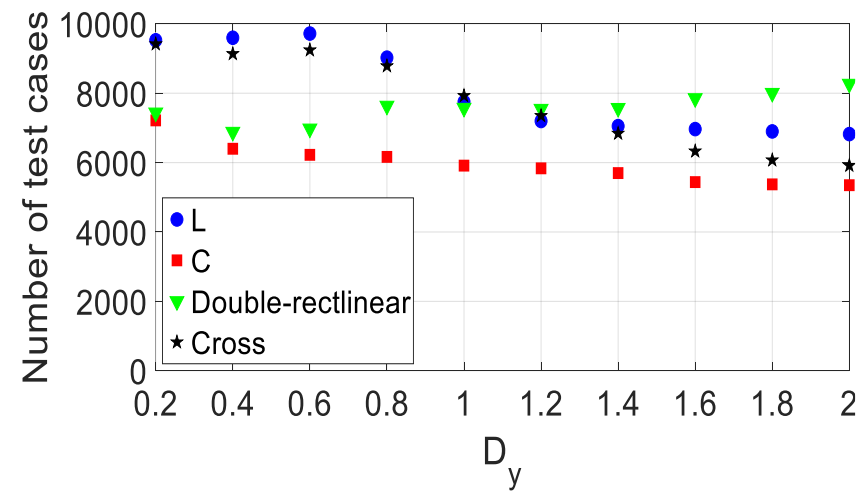

Fig. 4. Numbers of occurrence of secondary peaks (10000 random tag positions) versus the synthetic aperture $\boldsymbol{D}_{\boldsymbol{y}}$ for different trajectories.

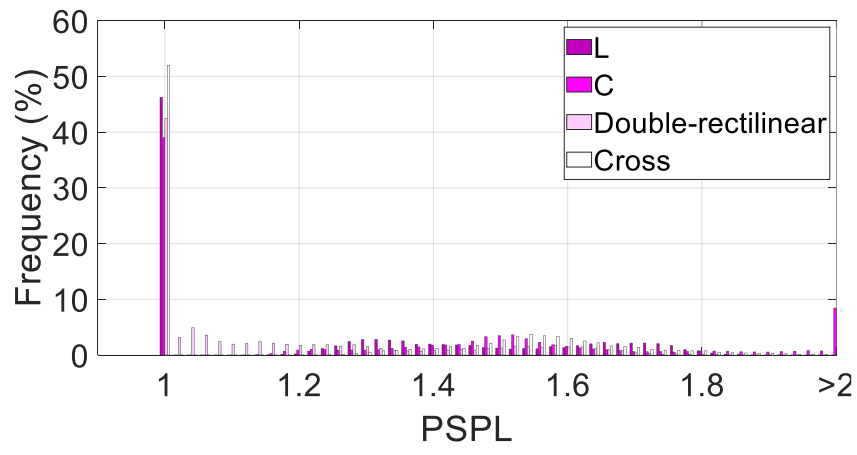

(b)

Fig. 5. Histograms (a) of the primary peak $C_{\max }$ and (b) of the Peak-to-Secondary-Peak-Level PSPL of the matching function for the L-shape trajectory (purple bars), the C-shape trajectory (pink bars), the double straight-line trajectory (light-pink bars) and the Cross-shape (white bars), when $D_{y}=2 \mathrm{~m}$.

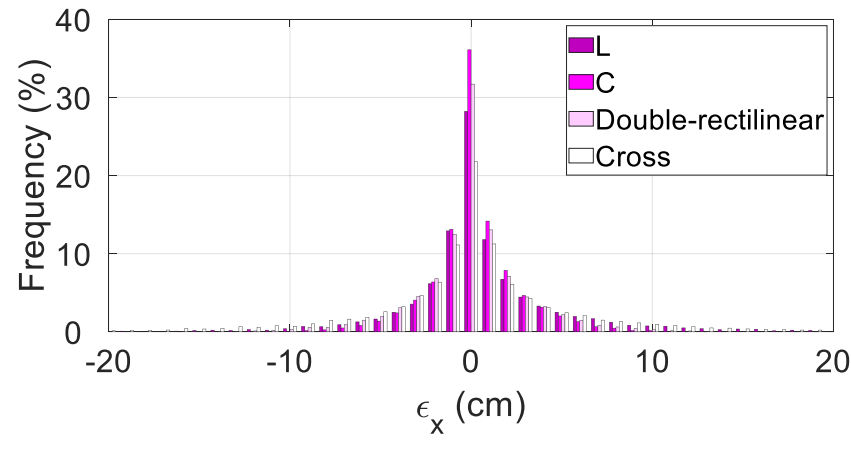

(a)

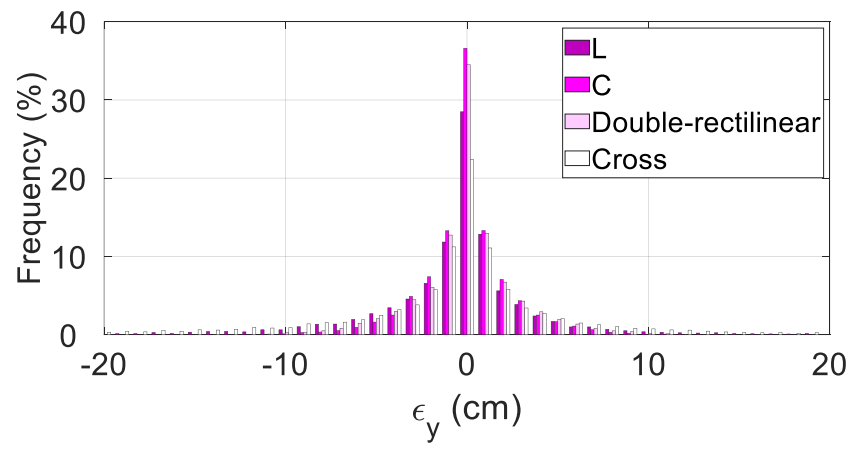

(b)

Fig. 6. Histograms of the localization error (a) on the $x$-coordinate $\epsilon_{x}$ and (b) on the $y$-coordinate $\epsilon_{y}$ for the L-shape trajectory (purple bars), the C-shape trajectory (pink bars), the double straight-line trajectory (light-pink bars) and the Cross-shape (white bars), when $D_{y}=2 \mathrm{~m}$. 


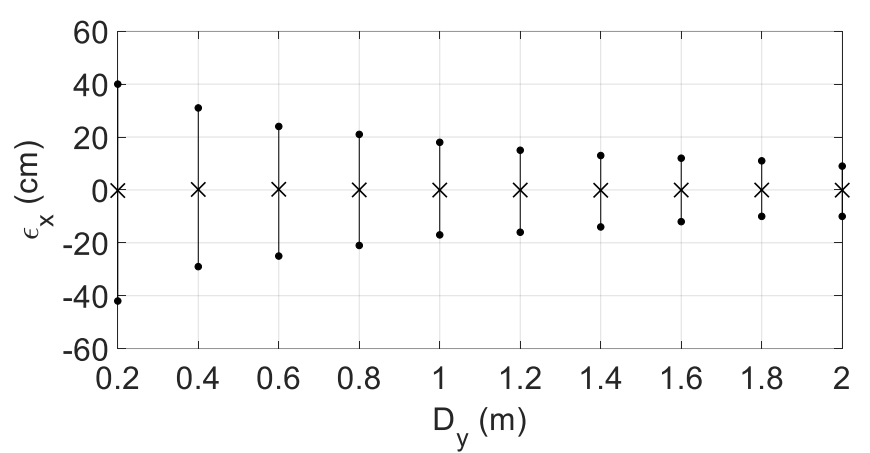

(a)

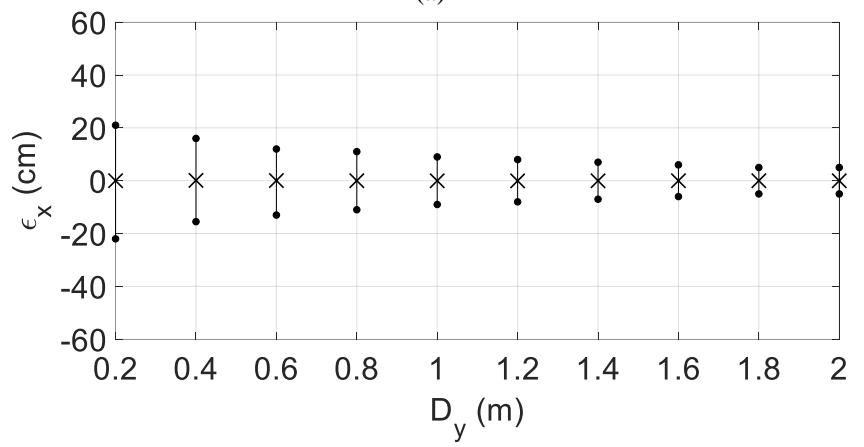

(c)

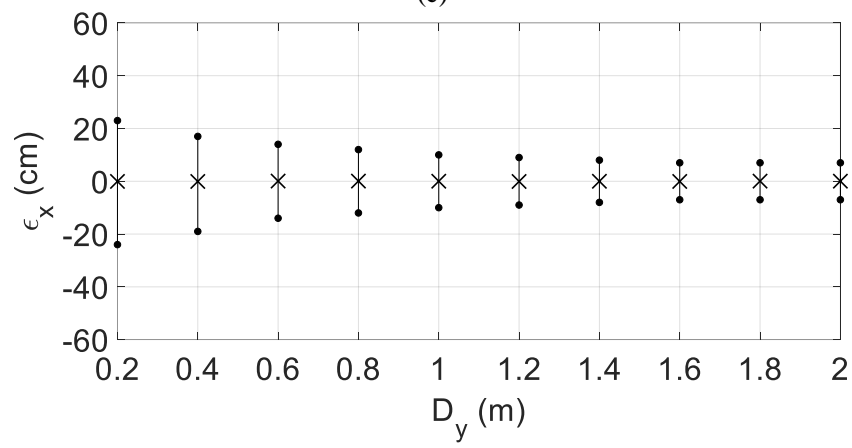

(e)

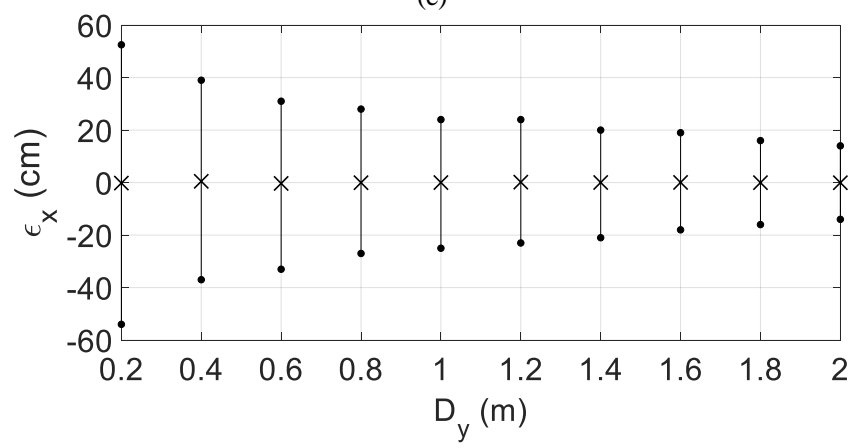

(g)

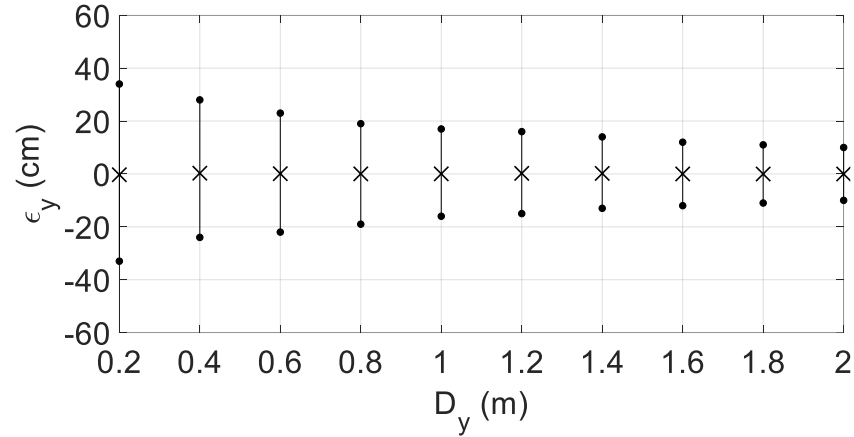

(b)

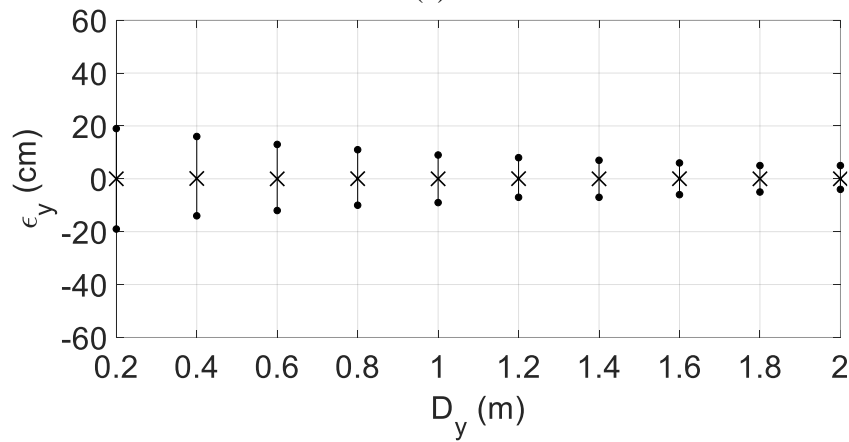

(d)

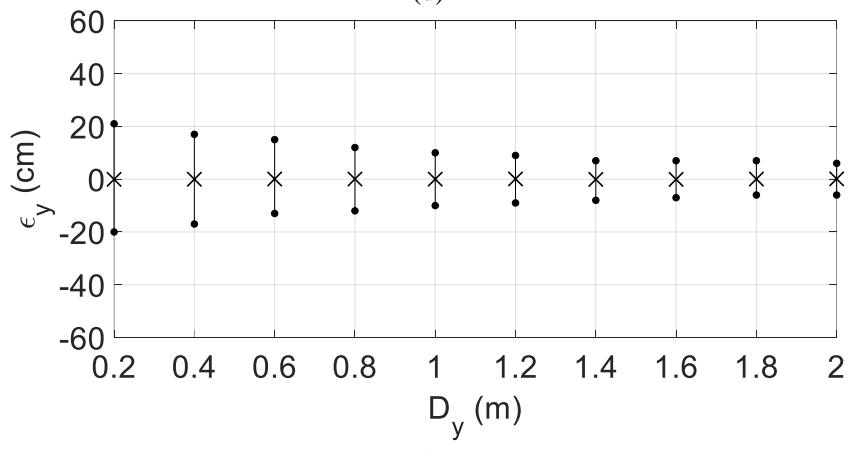

(f)

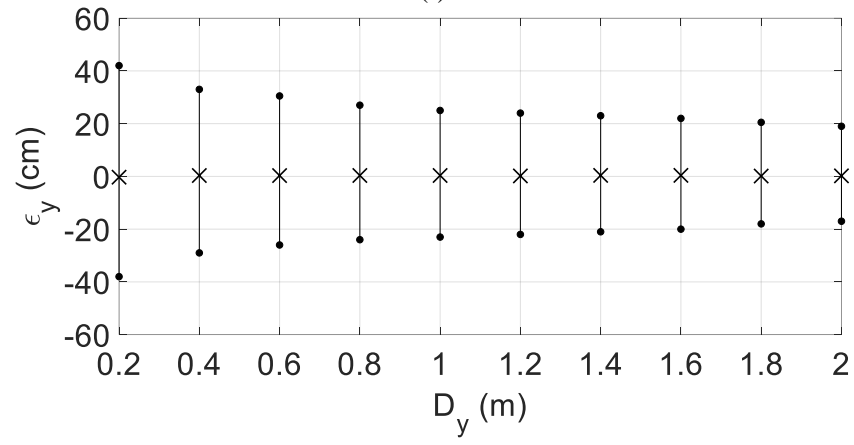

(h)

Fig. 7. Mean value and probabilistically symmetric $95 \%$ coverage interval of the localization error on the $x$-coordinate $\epsilon_{x}$ (a) for the L-shape trajectory, (c) the C-shape trajectory, (e) the double straight-line trajectory, (g) for the Cross-shape trajectory and on the $y$-coordinate $\epsilon_{y}$ (b) for the L-shape trajectory, (d) the C-shape trajectory, (f) the double straight-line trajectory, (h) for the Cross-shape trajectory.

Fig. 7 shows the mean values of the error distributions together with the probabilistically symmetric $95 \%$ coverage interval, by varying the $D_{y}$ synthetic aperture.

The C-shape trajectory has the best distribution of the PSPL parameter, namely the higher values, and this reflects on the localization error histograms which exhibits the highest frequency of small errors over both the $x$ - and the $y$ - coordinates. On the contrary, the Cross-shape trajectory exhibits the worst distribution of the PSPL parameter and consequently the highest localization error. This result suggests that the most significant parameter is the peak value of the matching function. Indeed, it is important to have a low PSPL to improve the localization performance. In Fig. 7, it appears that the probabilistically symmetric $95 \%$ coverage interval 
decreases if increasing the synthetic aperture $D_{y}$, as expected. The C-shape and the double straight-line trajectories show nearly equal performance. In fact, the results are much better than with the other considered trajectories. The Cross-shape trajectory shows different performance from the L-shape trajectory even if they have the same synthetic apertures. This is mainly due to the different trajectory shape.

The numerical analysis has been extended, by considering further multiple trajectories, with arbitrary shapes and different synthetic apertures, like those illustrated in Fig. 8. We refer to them as trajectory $T_{1}$ (circle blue markers), trajectory $T_{2}$ (square red markers) and trajectory $T_{3}$ (triangle green markers). All of them are combinations of two trajectory portions with the reader antenna at a height of $z_{n}=0.8 \mathrm{~m}$. The trajectories parameters are described in Table II. All of them meet the sampling condition (6). We also add the results of the multiple trajectory derived from the combination of trajectories $T_{1}, T_{2}$ and $T_{3}$.

The histograms of both the primary peak $C_{\max }$ (Fig. 9a) and the Peak-to-Secondary-Peak-Level PSPL (Fig. 9b) are represented in Fig. 9 for the trajectories of Fig. 8, still considering 10000 random tag positions. The histograms of the localization error for the $x$ - (Fig. 10a) and the $y$-coordinate (Fig. 10b) is represented in Fig. 10, while the corresponding the probabilistically symmetric $95 \%$ coverage intervals are shown in Fig. 11. The trajectory $T_{1}$ includes the higher number of test cases with low $C_{\max }$ values and the largest number of test cases with the PSPL parameter close to 1. Consequently, the corresponding localization error is the largest. The trajectory $\mathrm{T}_{2}$ and the trajectory $\mathrm{T}_{3}$ exhibit similar performance in terms of $C_{\text {max }}$, but $\mathrm{T}_{3}$ exhibits a better distribution of the PSPL parameter, with higher values. Thus, the associated localization error is lower. From these simulations, it is apparent that when the trajectory shape is arbitrary and complex, the total trajectory length is not the main parameter to derive the localization performance, differently from the conventional SAR method with straight linear trajectory, where the trajectory length is the main parameter to determine the system performance [55]. Indeed, the trajectory $T_{2}$ with $D_{\text {tot }}=3.91 \mathrm{~m}$ performs better than $T_{1}$ with $D_{\text {tot }}=4.45 \mathrm{~m}$.

For comparison, we also consider the case in which all the trajectories $T_{1}, T_{2}$ and $T_{3}$ are considered together. In this case,

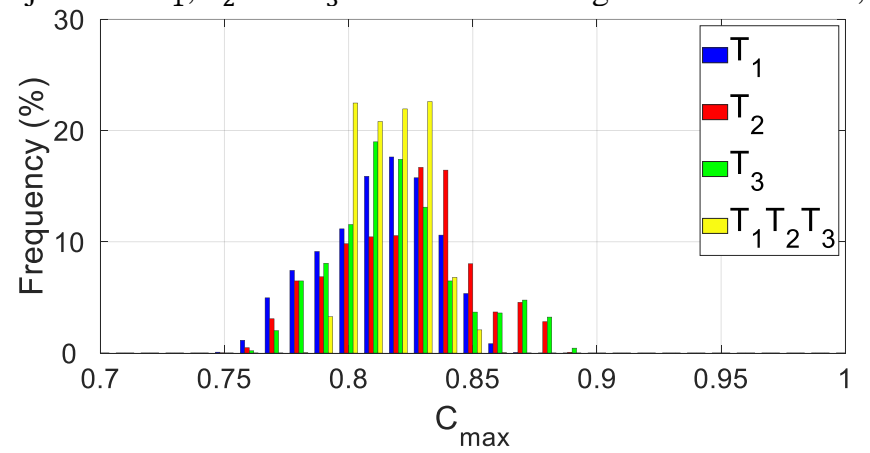

(a) the advantage of the joint process is evident from the localization error distribution (yellow bar in Fig. 10). As a result, the central $95 \%$ range of the localization error reduces to $6 \mathrm{~cm}$ and $8 \mathrm{~cm}$ for the $x$ - and the $y$-coordinate, respectively.

TABle II. PARAMETERS OF THE DifFerent TRAJECTORIES EMPLOYED IN THE NUMERICAL ANALYSIS.

\begin{tabular}{|l|l|l|l|l|}
\hline Trajectory & $T_{1}$ & $T_{2}$ & $T_{3}$ & $T_{1} T_{2} T_{3}$ \\
\hline $\boldsymbol{D}_{\boldsymbol{x}}(\mathbf{m})$ & 2.00 & 2.50 & 2.28 & 2.78 \\
\hline $\boldsymbol{D}_{\boldsymbol{y}}(\mathbf{m})$ & 2.00 & 1.00 & 2.00 & 3.00 \\
\hline $\boldsymbol{D}_{\text {tot }}(\mathbf{m})$ & 4.45 & 3.91 & 5.50 & 18.04 \\
\hline$\Delta(\mathbf{c m})$ & 5.9 & 5.1 & 5.7 & 7.2 \\
\hline
\end{tabular}

\section{B. Combination of consecutive estimated tag positions}

As aforementioned, when consecutive tag position estimates are available, different criteria can be adopted to determine the lowest uncertainty estimates. We consider again the set of multiple trajectories of Fig. 8, and Monte Carlo simulations are carried out by considering 10000 random tag positions. Fig. 12 represents the histograms of the localization errors along the $x$ (Fig. 12a) and the $y$-axis (Fig. 12b), respectively, by using the criteria described in Section II, while the corresponding mean values with the central 95\% range are in Fig. 13.

We add also the result of the processing of all phase data collected over all trajectories, i.e. $T_{1}, T_{2}$ and $T_{3}$.

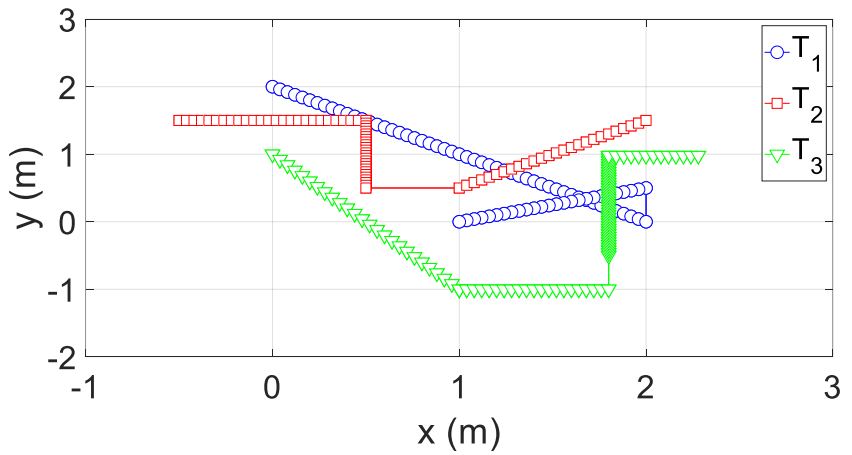

Fig. 8. Example of the set of multiple trajectories employed in the numerical analysis: trajectory $\boldsymbol{T}_{1}$ (circle blue markers), trajectory $\boldsymbol{T}_{2}$ (square red markers) and trajectory $\boldsymbol{T}_{\mathbf{3}}$ (triangle green markers).

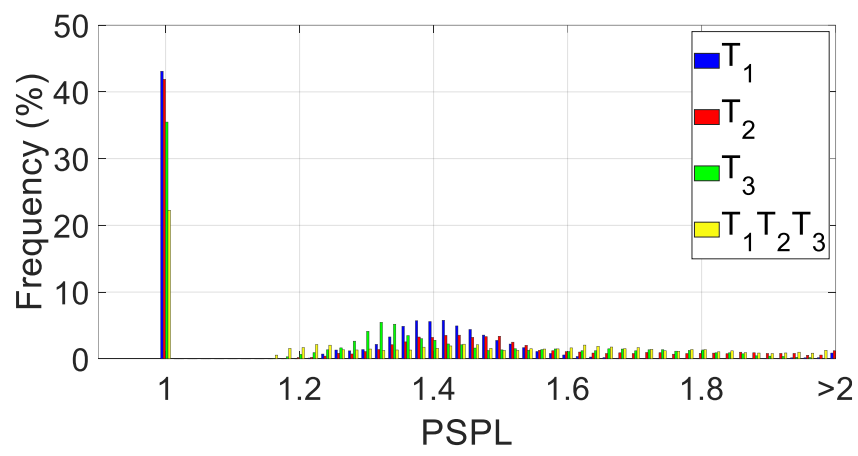

(b)

Fig. 9. Histograms (a) of the primary peak $C_{\max }$ and (b) of the Peak-to-Secondary-Peak-Level PSPL of the matching function the multiple trajectories of Fig. 8. 


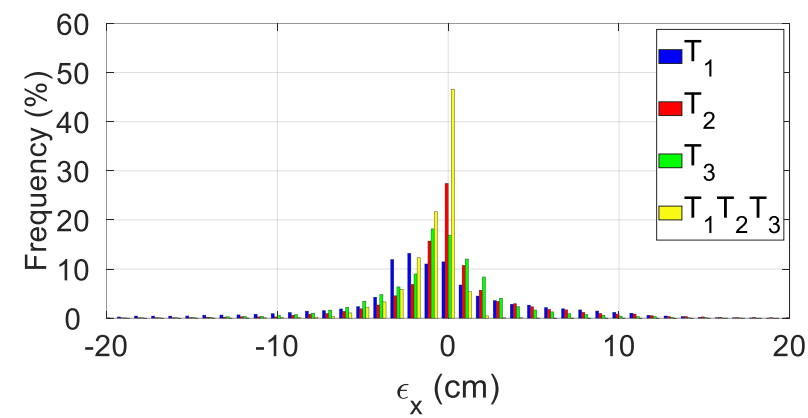

(a)

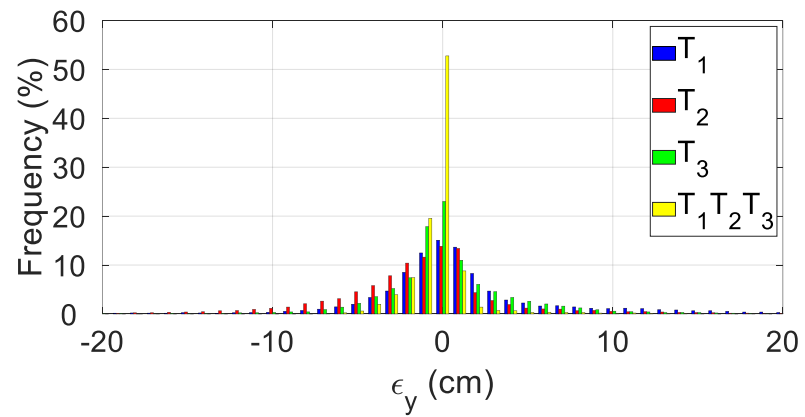

(b)

Fig. 10. Histograms of the localization error (a) on the $x$-coordinate $\epsilon_{x}$ and (b) on the $y$-coordinate $\epsilon_{y}$ for the multiple trajectories of Fig. 8.

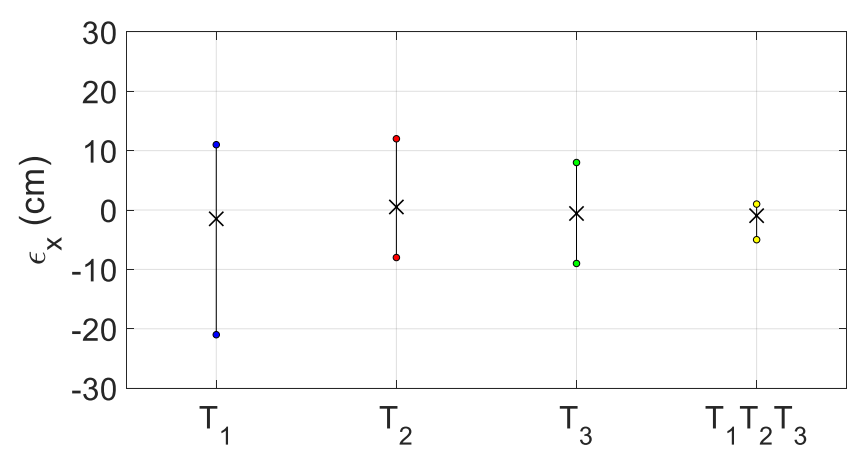

(a)

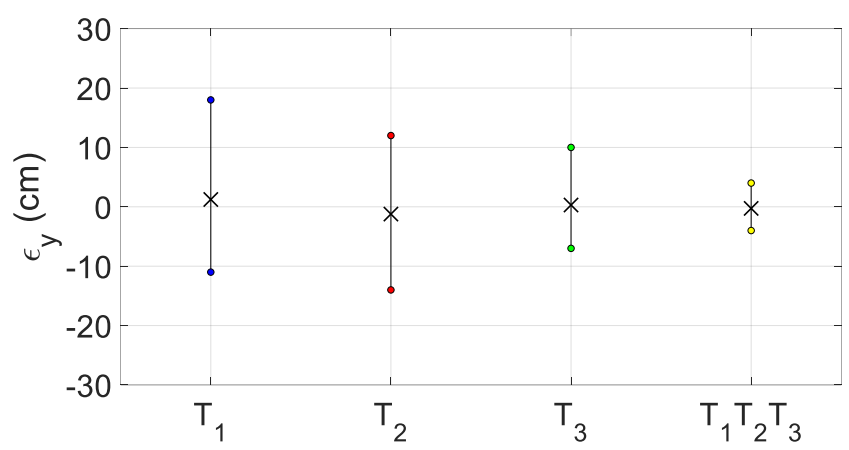

(b)

Fig. 11. Mean value and probabilistically symmetric $95 \%$ coverage interval of the localization error (a) on the $x$-coordinate $\epsilon_{x}$ and (b) on the $y$-coordinate $\epsilon_{y}$ for the multiple trajectories of Fig. 8.

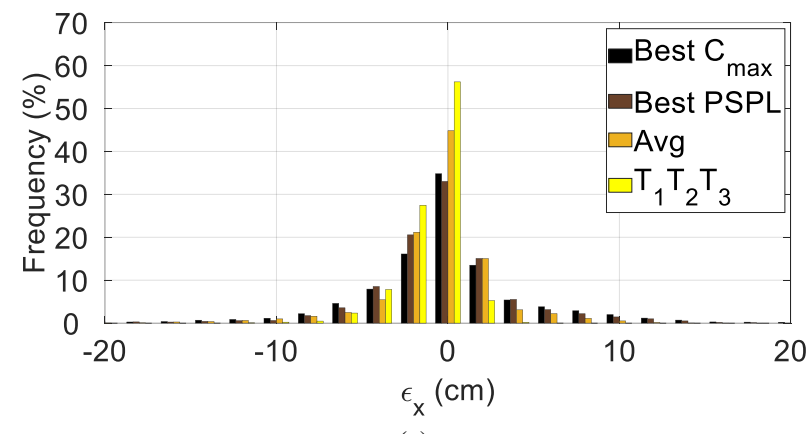

(a)

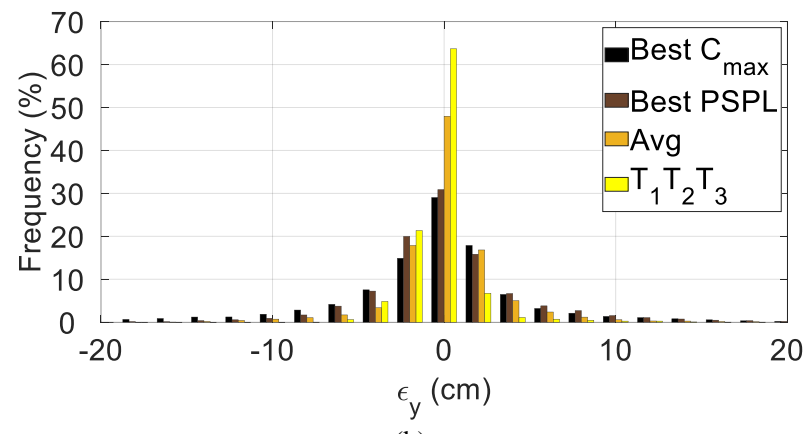

(b)

Fig. 12. Histograms of the localization error (a) on the $x$-coordinate $\varepsilon_{x}$ and (b) on the $y$-coordinate $\varepsilon_{y}$ for the different estimation criteria.

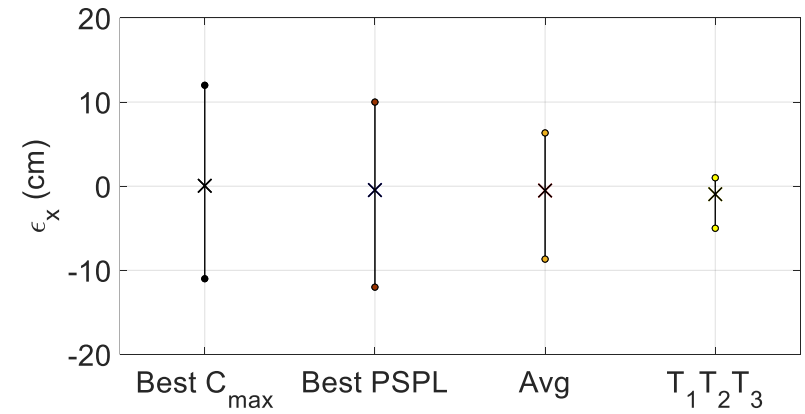

(a)

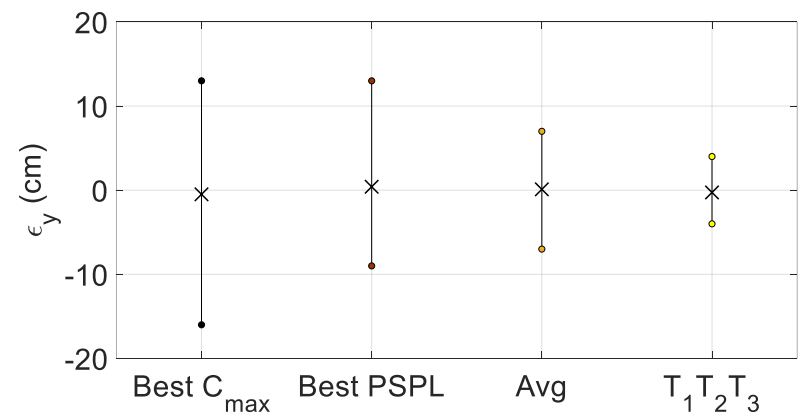

(b)

Fig. 13 Mean value and probabilistically symmetric $95 \%$ coverage interval of the localization error (a) on the $x$-coordinate $\varepsilon_{x}$ and (b) on the $y$-coordinate $\varepsilon_{y}$ for the different estimation criteria.

Among the considered criteria, the average operation among consecutive estimated tag positions (14) minimizes the localization errors with respect to the criteria based on the best value of $C_{\max }$ or PSPL.
However, the results obtained from the data collected over all trajectories outperform the other criteria. Obviously, the latter can be applied only if the sampling theorem is satisfied. 


\section{EXPERIMENTAL ANALYSIS}

\section{A. Experimental Setup and Trajectory Measurements}

The experimental campaign to analyse the performance of the proposed localisation method were conducted in the premises of the University of Trento, by employing commercial RFID hardware and a robotic wheeled walker.

The robot was equipped with the Impinj Speedway Revolution R420 UHF-RFID reader and the WANTENNAX019 antenna by C.A.E.N. RFID (Fig. 14). It is powered by a battery installed on the robot. The reader input power was set to $P_{T X}=27 \mathrm{dBm}$, in order to satisfy the limit on the Effective Isotropic Radiative Power (EIRP), since the antenna gain is $\mathrm{G}=8.5 \mathrm{dBc}$ and the cable losses amount to 1.5 dB. $6 \times 9$ commercial UHF RFID inlay tags were deployed over an area of $40 \mathrm{~m}^{2}$, as shown in Fig. 15. Different types of tags were used to check the method effectiveness regardless of the tag characteristics. Different tag orientations were also tested, since the reader antenna radiates a circularly polarized field. Tags on odd (even) rows are aligned along the $x$-direction ( $y$ direction). The tag main parameters are summarized in Table III.

An OptiTrack motion capture system was used to measure the trajectory of the reader antenna with high accuracy (which is a basic requirement to test the accuracy of the proposed SARbased measurement method). OptiTrack system is equipped with 14 Prime 13 smart cameras (Fig. 15) able to stream data to a central server with a declared latency of $4.2 \mathrm{~ms}$ and negligible jitter. The OptiTrack system is able to measure the position of ad-hoc markers in a known reference frame (as depicted in Fig. 15). The declared positioning and orientation standard uncertainties of the vision system are below $1 \mathrm{~mm}$ and below $0.1^{\circ}$, respectively, in a room of about $8 \mathrm{~m} \times 8 \mathrm{~m} \times 2 \mathrm{~m}$ [56]. The cameras frame rate was set to $120 \mathrm{~Hz}$ and 18 markers were placed on the robotic walker to reconstruct its rigid body posture. More precisely, six markers were placed on each handle, one marker was placed on each rear wheel and three markers were placed on the RFID antenna to keep track of its position and orientation, as shown in Fig. 14. This large number of markers enables an effective position tracking even when some of the markers are occluded.

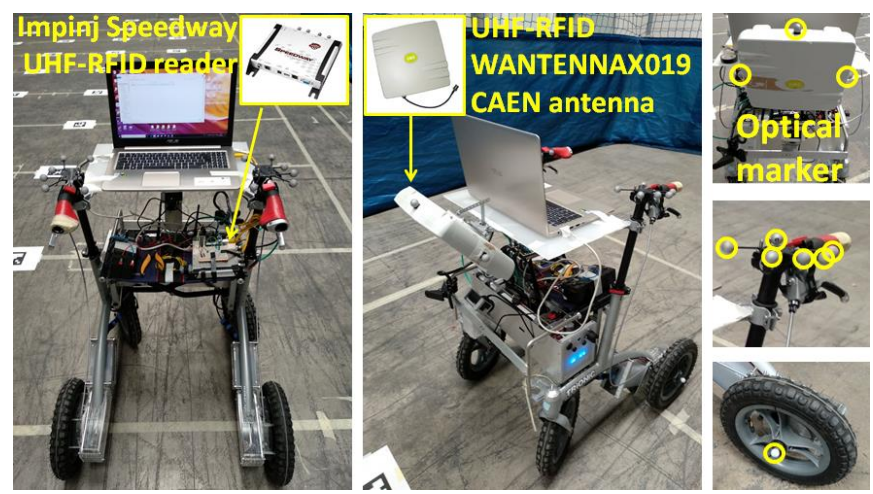

Fig. 14. Robotic wheeled walker equipped with the Impinj Speedway Revolution R420 UHF-RFID reader, the UHF-RFID WANTENNAX019 antenna by C.A.E.N. RFID and optical markers.

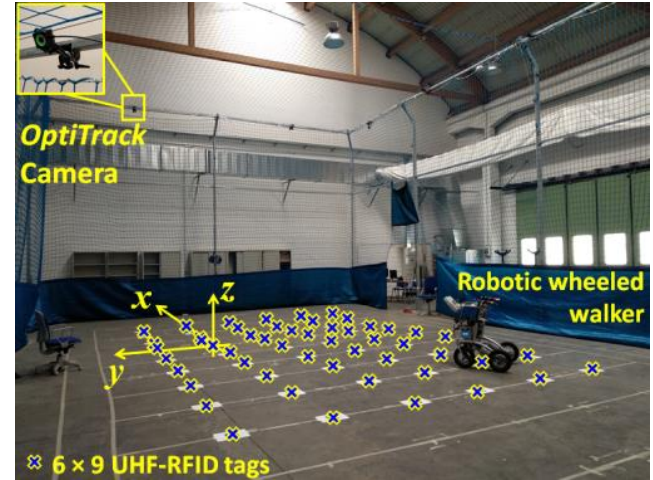

Fig. 15. Indoor scenario with $6 \times 9$ commercial UHF-RFID tags deployed in an area of $40 \mathrm{~m}^{2}$ and the OptiTrack motion capture system with 14 Prime13 smart cameras.

TABLE III

MAIN PARAMETERS OF THE TAGS EMPLOYED IN THE EXPERIMENTAL SETUP.

\begin{tabular}{|l|l|l|l|}
\hline Tag & Tag size & Chip & $\begin{array}{l}\text { Chip read } \\
\text { sensitivity }\end{array}$ \\
\hline \begin{tabular}{l} 
Easy RFID Clepsidra \\
\hline $\begin{array}{l}\text { Easy RFID Fashion } \\
\text { Easy RFID Garbage } \\
\text { LAB ID UH3D40 }\end{array}$
\end{tabular} & $70 \times 50 \mathrm{~mm}^{2}$ & MZ-6 & $-22.1 \mathrm{dBm}$ \\
\hline $\begin{array}{l}\text { LAB ID UH331 } \\
\text { LABID UH106 }\end{array}$ & $95 \times 10 \mathrm{~mm}^{2}$ & $\begin{array}{l}\text { NXP } \\
\text { UCODE-7 }\end{array}$ & $-21 \mathrm{dBm}$ \\
\hline Smartrac Dogbone & $85 \times 40 \mathrm{~mm}^{2}$ & MZ-4 & $-19.5 \mathrm{dBm}$ \\
\hline
\end{tabular}

The data collected by the RFID and the OptiTrack systems are time-aligned to minimize the positioning estimation uncertainty due to possible lags between the two system. The reference vision system was also used to measure, with negligible uncertainty, the ground truth positions of the UHFRFID tags placed on the floor. This is essential to evaluate the performance of the proposed SAR-based localization method. For this reason, some markers were also placed on top of the RFID tags. An example of a robotic walker path along with the position of the tags is shown in Fig. 16. In particular, the walker moves freely in the area and the tag \#90172 (green circular marker) placed at $\mathbf{p}_{\text {tag }}=[-1.77,-0.03] \mathrm{m}$ is detected along different paths. We consider the trajectory \#A formed by two nearby parallel portions (blue circular markers) and the trajectory \#B composed by two crossed paths (red square markers). The multiple trajectory parameters are summarized in Table IV.

\section{B. Localization performance}

The sequence of phase data relative to tag \#90172 is represented in Fig. 17, when the walker moves over the multitrajectory \#A (circle markers) and the multi-trajectory \#B (square markers), respectively. 


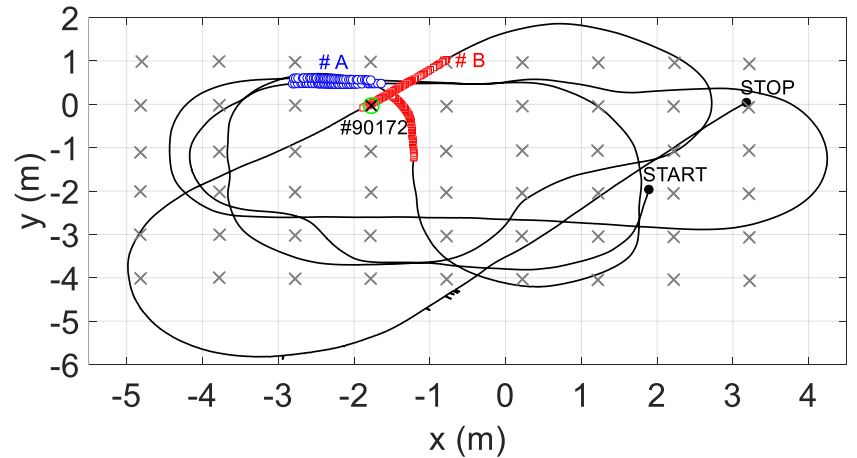

Fig. 16. Example of the reader antenna trajectory measured by the visionbased system. The tag positions (cross markers) are shown as well. The two multiple trajectories along which the tag \#90172 (green circular marker) is detected are reported with coloured markers.

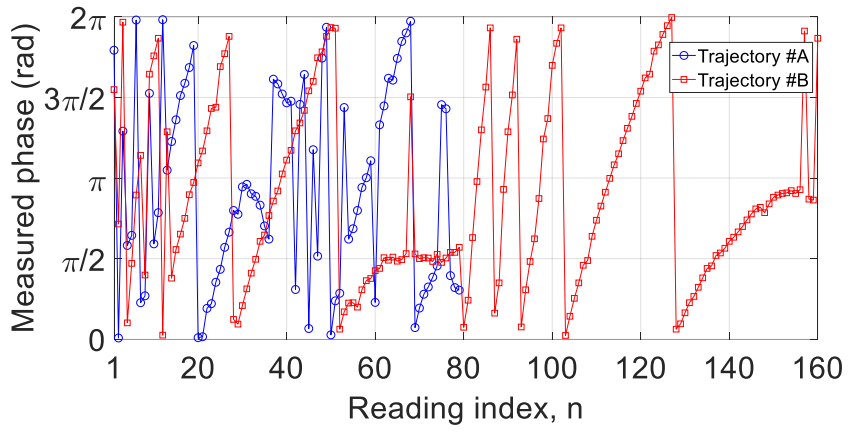

Fig. 17. Measured phase sequence with respect to the reading index $\boldsymbol{n}$, when the tag \#90172 is detected over trajectory \#A (circle markers) and trajectory \#B (square markers) of Fig. 16.

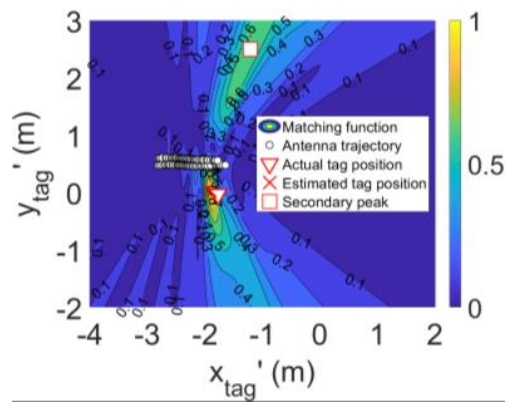

(a)

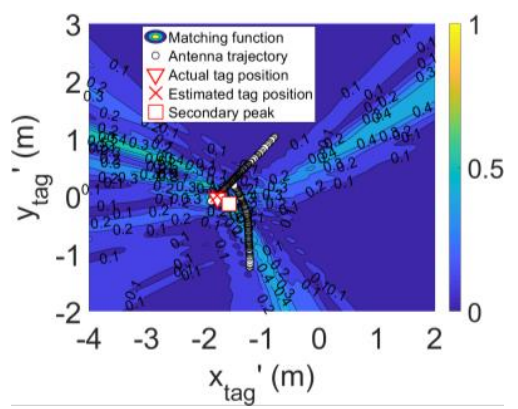

(b)

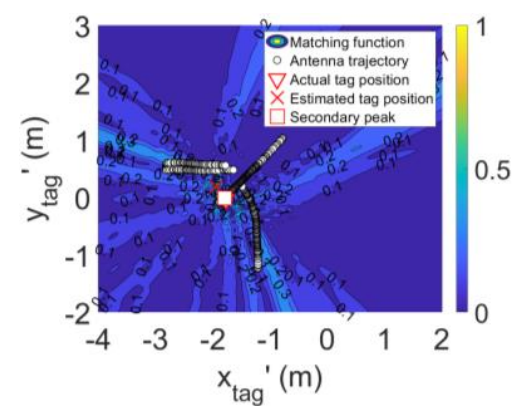

(c)

Fig. 18. Matching function and the corresponding estimated tag position by applying the SAR-based method for the different measured trajectories of Fig. 16: (a) trajectory \#A, (b) trajectory \#B, and (c) trajectory \#AB.

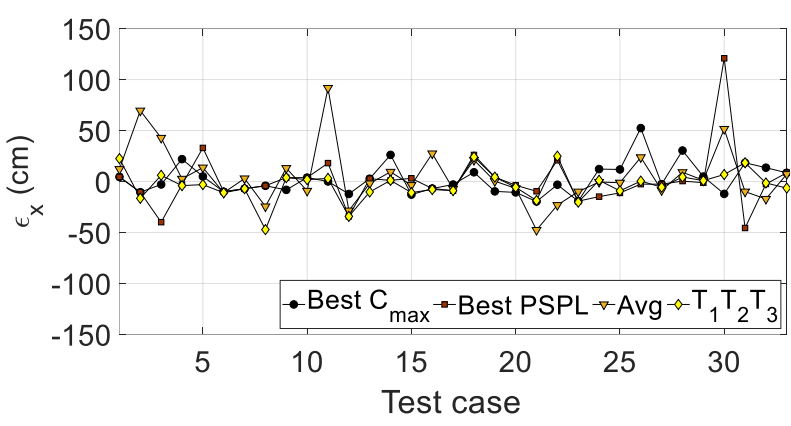

(a)

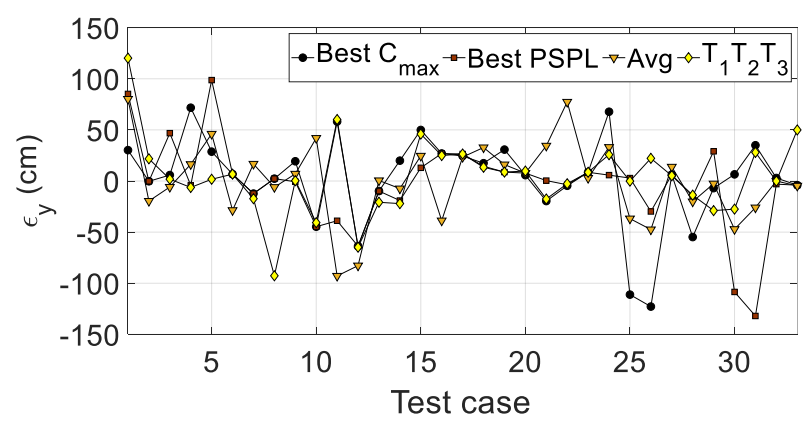

(b)

Fig. 19. Measured localization error (a) on the $x$-coordinate $\epsilon_{x}$ and (b) on the $y$-coordinate $\epsilon_{y}$ for the different estimation criteria.

Table IV. Parameters of the Measured Trajectories During the DETECTION OF TAG \#90172 PLACED AT $\mathbf{p}_{\mathbf{t a g}}=[\mathbf{- 1 . 7 7}, \mathbf{- 0 . 0 3}] \mathbf{m}$.

\begin{tabular}{|l|l|l|l|}
\hline Trajectory & \#A & \#B & \#AB \\
\hline$N_{r}$ & 79 & 160 & 239 \\
\hline$D_{x}(\mathrm{~m})$ & 1.16 & 1.11 & 2.03 \\
\hline$D_{y}(\mathrm{~m})$ & 0.15 & 2.27 & 2.27 \\
\hline$D_{\text {tot }}(\mathrm{m})$ & 3.38 & 4.17 & 9.43 \\
\hline$\Delta(\mathrm{cm})$ & 4.3 & 2.6 & 4.0 \\
\hline$C_{\max }$ & 0.90 & 0.71 & 0.65 \\
\hline$C_{s p}$ & 0.68 & 0.63 & 0.64 \\
\hline$P S P L$ & 1.32 & 1.13 & 1.02 \\
\hline$\widehat{\boldsymbol{x}}_{\text {tag }}$ & -1.91 & -1.78 & -1.94 \\
\hline$\widehat{\boldsymbol{y}}_{\text {tag }}$ & 0.03 & 0.01 & 0.19 \\
\hline$x_{s p}$ & -1.21 & -1.57 & -1.79 \\
\hline$y_{s p}$ & 2.50 & -0.13 & -0.01 \\
\hline
\end{tabular}

Some unpredictable phase jumps are clearly visible as reported in the reader datasheet [54].

The corresponding matching functions and its parameters are reported in Fig. 18 and in Table IV, respectively. Multitrajectory \#B is estimated with a lower localization uncertainty than trajectory \#A. Since two consecutive estimates are available, the best estimation criterion described in Section II was used. In this case, $\widehat{\mathbf{p}}_{\text {tag-best1 }}=\widehat{\mathbf{p}}_{\text {tag-best2 }}=$ $[-1.91,0.03] \mathrm{m}, \widehat{\mathbf{p}}_{\text {tag-avg }}=[-1.85,0.02] \mathrm{m}$, while the result of the combination of trajectory \#A and \#B determines $\widehat{\mathbf{p}}_{\text {tag-AB }}=[-1.94,0.19] \mathrm{m}$.

Unlike the simulation results, it seems that in this case the criterion based on the average between the two estimated position yields the best performance. However, an extensive measurement campaign has been carried out to determine the overall performance. The measured localization errors are represented in Fig. 19, for 33 test cases with three multiple trajectories having $D_{x}>0.3 \mathrm{~m}$ and $D_{y}>0.3 \mathrm{~m}$. Three estimated positions are available for each tag and the different estimation criteria are compared. According to the simulated 
analysis, the best choice is to combine the data collected along the three trajectories altogether (to compose the multiple trajectory $T_{1} T_{2} T_{3}$ ).

\section{CONCLUSION}

The application of the Synthetic Aperture Radar localization method for indoor positioning of UHF-RFID tags has been presented, when a robot equipped with reader antenna moves over multiple trajectories (which may not be contiguous in time). The phase data collected during multiple paths can be profitably employed to improve the localization accuracy, provided that the sampling theorem was satisfied. Performance is not only dependent on the trajectory length as in classical SAR methods with straight linear trajectories, but also on the trajectory shape with respect to the tag position.

Besides, the availability of multiple tag position estimates during consecutive inventory rounds can be properly combined to reduce the localization uncertainty. Different estimation criteria were investigated through the paper: the best matching function peak, the best peak-to-secondary-peak level, the average of consecutive estimates and the combination of the phase data collected during all the available trajectories.

The proposed localization method has been firstly investigated through a numerical analysis by considering different configurations of the multiple apertures and different sources of measurement uncertainty. Then, it has been validated with commercial RFID hardware and a robotic wheeled walker in an indoor scenario, by employing different types of tags. Both the numerical and the experimental analysis showed that the best way to reduce the localization uncertainty is to combine the data collected along all available trajectories, even if distant in time, provided that sampling theorem requirement was met. If this is not possible, it is convenient to use the average of consecutive tag position estimates.

\section{REFERENCES}

[1] F. Zafari, A. Gkelias, and K. K. Leung, "A Survey of Indoor Localization Systems and Technologies," IEEE Communications Surveys \& Tutorials, vol. 21, no. 3, pp. 2568-2599, thirdquarter 2019.

[2] F. Endres, J. Hess, J. Sturm, D. Cremers, and W. Burgard, "3-D Mapping With an RGB-D Camera," IEEE Transactions on Robotics, vol. 30, no. 1, pp. 177-187, Feb. 2014.

[3] F. Kallasi, D. L. Rizzini, and S. Caselli, "Fast Keypoint Features From Laser Scanner for Robot Localization and Mapping," IEEE Robotics and Automation Letters, vol. 1, no. 1, pp. 176-183, Jan. 2016.

[4] R. Gutierrez-Osuna, J. A. Janet, and R. C. Luo, "Modeling of ultrasonic range sensors for localization of autonomous mobile robots," IEEE Transactions on Industrial Electronics, vol. 45, no. 4, pp. 654-662, Aug. 1998.

[5] L. Zhang and H. Wang, "3D-WiFi: 3D Localization With Commodity WiFi," IEEE Sensors Journal, vol. 19, no. 13, pp. 5141-5152, 1 July1, 2019.

[6] P. Barsocchi, A. Crivello, M. Girolami, F. Mavilia, and F. Palumbo, "Occupancy detection by multi-power bluetooth low energy beaconing", 2017 International Conference on Indoor Positioning and Indoor Navigation (IPIN), Sapporo, 2017, pp. 1-6.

[7] A. Cazzorla, G. De Angelis, A. Moschitta, M. Dionigi, F. Alimenti, and P. Carbone, "A 5.6-GHz UWB Position Measurement System", IEEE Transactions on Instrumentation and Measurement, vol. 62, no. 3, pp. 675-683, March 2013.
[8] V. Pasku, A. De Angelis, G. De Angelis, A. Moschitta, and P. Carbone, "Magnetic Field Analysis for 3-D Positioning Applications", IEEE Transactions on Instrumentation and Measurement, vol. 66, no. 5, pp. 935-943, May 2017.

[9] W. Lv, Y. Kang, and J. Qin, "Indoor Localization for Skid-Steering Mobile Robot by Fusing Encoder, Gyroscope, and Magnetometer," IEEE Transactions on Systems, Man, and Cybernetics: Systems, vol. 49, no. 6, pp. 1241-1253, June 2019.

[10] C. Li, L. Mo and D. Zhang, "Review on UHF RFID Localization Methods," IEEE Journal of Radio Frequency Identification, vol. 3, no. 4, pp. 205-215, Dec. 2019.

[11] P. Nepa et al., "I-READ 4.0: Internet-of-READers for an efficient asset management in large warehouses with high stock rotation index," 2019 IEEE 5th International forum on Research and Technology for Society and Industry (RTSI), Florence, Italy, 2019, pp. 67-72.

[12] J. Zhang, Y. Lyu, J. Patton, S. C. G. Periaswamy and T. Roppel, "BFVP: A Probabilistic UHF RFID Tag Localization Algorithm Using Bayesian Filter and a Variable Power RFID Model," IEEE Transactions on Industrial Electronics, vol. 65, no. 10, pp. 8250-8259, Oct. 2018.

[13] L. Shangguan, Z. Yang, A. X. Liu, Z. Zhou, and Y. Liu, "STPP: SpatialTemporal Phase Profiling-Based Method for Relative RFID Tag Localization ", IEEE/ACM Transactions on Networking, vol. 25, no. 1, pp. 596-609, Feb. 2017.

[14] G. Roussos, "Enabling RFID in retail", Computer, vol. 39, no. 3, pp. 2530, March 2006.

[15] Fornes, Feb 28, 2017, "Robots Will Be In Retail Stores Sooner Than You Think" [Online]. Available: https://www.forbes.com/sites/nikkibaird/2017/02/28/robots-in-retailstores-closer-than-you-think/\#1edac3ea5d96.

[16] A. Raptopoulos, T. Yioultsis, and A. G. Dimitriou, "Particle Filter Object Tracking by a Handheld UHF RFID Reader," 2019 IEEE International Conference on RFID Technology and Applications (RFID-TA), Pisa, Italy, 2019, pp. 342-347.

[17] H. Farhat, P. Iliev, P. Marriage, and N. Rolland, "An Added Value Alternative to RAIN RFID Items Characterization in Retail," IEEE Access, vol. 6, pp. 32430-32439, 2018.

[18] V. Magnago et al., "Ranging-Free UHF-RFID Robot Positioning Through Phase Measurements of Passive Tags," IEEE Transactions on Instrumentation and Measurement, vol. 69, no. 5, pp. 2408-2418, May 2020.

[19] K. Nur, M. Morenza-Cinos, A. Carreras, and R. Pous, "Projection of RFID-Obtained Product Information on a Retail Stores Indoor Panoramas", IEEE Intelligent Systems, vol. 30, no. 6, pp. 30-37, Nov.Dec. 2015.

[20] C. R. Medeiros, J. R. Costa, and C. A. Fernandes, "RFID Reader Antennas for Tag Detection in Self-Confined Volumes at UHF", IEEE Antennas and Propagation Magazine, vol. 53, no. 2, pp. 39-50, April 2011.

[21] A. Michel, R. Caso, A. Buffi and P. Nepa, "Multifunctional modular antenna for near-field ultra-high frequency radio frequency identification readers," IET Microwaves, Antennas \& Propagation, vol. 10, no. 8, pp. 843-849, 56 2016, doi: 10.1049/iet-map.2015.0632.

[22] J. Lai et al., "TagSort: Accurate Relative Localization Exploring RFID Phase Spectrum Matching for Internet of Things," IEEE Internet of Things Journal, vol. 7, no. 1, pp. 389-399, Jan. 2020.

[23] T. Hasler, M. Wölbitsch, M. Goller, and S. Walk, "Relative Tag Locations Based on Time-Differences in Read Events for Practical Applications," IEEE Journal of Radio Frequency Identification, vol. 4, no. 1, pp. 55-64, March 2020.

[24] Graham P. Eloy, "Steerable Phase Array Antenna RFID Tag Locater and Tracking System and Method", US 8,742,896 B2 patent, 2014.

[25] "xArray Rain RFID Gateway", Impinj, 2018. [Online]. Available: https://www.impinj.com/platform/connectivity/xarray/.

[26] RFID robot for automatic inventory: AdvanRobotTM https://www.keonn.com/systems/view-all-2/inventory-robots.html

[27] TORY: Efficient automated RFID Inventory https://www.metralabs.com/en/rfid-robot-tory/

[28] Tag Surveyor: Autonomous RFID Tag Tracking https://fetchrobotics.com/products-technology/datasurvey/tagsurveyor/

[29] A. Buffi, P. Nepa, and F. Lombardini, "A Phase-Based Technique for Localization of UHF-RFID Tags Moving on a Conveyor Belt: 
Performance Analysis and Test-Case Measurements," IEEE Sensors Journal, vol. 15, no. 1, pp. 387-396, Jan. 2015.

[30] Z. Zhang, Z. Lu, V. Saakian, X. Qin, Q. Chen, and L. Zheng, "Item-Level Indoor Localization With Passive UHF RFID Based on Tag Interaction Analysis," IEEE Transactions on Industrial Electronics, vol. 61, no. 4, pp. 2122-2135, April 2014.

[31] Y. Zhao, K. Liu, Y. Ma, Z. Gao, Y. Zang, and J. Teng, "Similarity Analysis-Based Indoor Localization Algorithm With Backscatter Information of Passive UHF RFID Tags," IEEE Sensors Journal, vol. 17, no. 1, pp. 185-193, 1 Jan.1, 2017.

[32] J. Wang and D. Katabi Dude, "Where's My Card?: RFID Positioning ThatWorks with Multipath and Non-Line of Sight," Proc. of ACM SIGCOMM, 2013.

[33] L. Yang, Q. Liu, J. Xu, J. Hu, and T. Song, "An Indoor RFID Location Algorithm Based on Support Vector Regression and Particle Swarm Optimization," 2018 IEEE 88th Vehicular Technology Conference (VTCFall), Chicago, IL, USA, 2018, pp. 1-6.

[34] C. Wang, Z. Shi, F. Wu, and J. Zhang, "An RFID indoor positioning system by using Particle Swarm Optimization-based Artificial Neural Network," 2016 International Conference on Audio, Language and Image Processing (ICALIP), Shanghai, 2016, pp. 738-742.

[35] Y. Ma, B. Wang, X. Gao, and W. Ning, "The Gray Analysis and Machine Learning for Device-Free Multitarget Localization in Passive UHF RFID Environments," IEEE Transactions on Industrial Informatics, vol. 16, no. 2, pp. 802-813, Feb. 2020.

[36] P. V. Nikitin, R. Martinez, S. Ramamurthy, H. Leland, G. Spiess, and K. V. S. Rao, "Phase based spatial identification of UHF RFID tags", 2010 IEEE International Conference on RFID (IEEE RFID 2010), Orlando, FL, 2010, pp. 102-109.

[37] D. Arnitz, K. Witrisal, and U. Muehlmann, "Multifrequency ContinuousWave Radar Approach to Ranging in Passive UHF RFID", IEEE Transactions on Microwave Theory and Techniques, vol. 57, no. 5, pp. 1398-1405, May 2009.

[38] S. Azzouzi, M. Cremer, U. Dettmar, R. Kronberger, and T. Knie, "New measurement results for the localization of UHF RFID transponders using an Angle of Arrival (AoA) approach", 2011 IEEE International Conference on RFID, Orlando, FL, 2011, pp. 91-97.

[39] M. Scherhäufl, M. Pichler and A. Stelzer, "Localization of passive UHF RFID tags based on inverse synthetic apertures," 2014 IEEE International Conference on RFID (IEEE RFID), Orlando, FL, 2014, pp. 82-88, doi: 10.1109/RFID.2014.6810716.

[40] A. Buffi, P. Nepa, and F. Lombardini, “A Phase-Based Technique for Localization of UHF-RFID Tags Moving on a Conveyor Belt: Performance Analysis and Test-Case Measurements," IEEE Sensors Journal, vol. 15, no. 1, pp. 387-396, Jan. 2015.

[41] A. Buffi, M. R. Pino, and P. Nepa, "Experimental Validation of a SARBased RFID Localization Technique Exploiting an Automated Handling System," IEEE Antennas and Wireless Propagation Letters, vol. 16, pp. 2795-2798, 2017.

[42] R. Miesen, F. Kirsch, and M. Vossiek, "UHF RFID Localization Based on Synthetic Apertures", IEEE Transactions on Automation Science and Engineering, vol. 10, no. 3, pp. 807-815, July 2013.

[43] A. Motroni et al., "SAR-Based Indoor Localization of UHF-RFID Tags via Mobile Robot," 2018 International Conference on Indoor Positioning and Indoor Navigation (IPIN), Nantes, 2018, pp. 1-8.

[44] J. Liu, F. Zhu, Y. Wang, X. Wang, Q. Pan, and L. Chen, "RF-scanner: Shelf scanning with robot-assisted RFID systems," IEEE INFOCOM 2017 - IEEE Conference on Computer Communications, Atlanta, GA, 2017, pp. 1-9.

[45] A. Buffi, A. Motroni, P. Nepa, B. Tellini, and R. Cioni, "A SAR-based Measurement Method for Passive-Tag Positioning with a Flying UHFRFID Reader", IEEE Transactions on Instrumentation and Measurement, vol. 68, no. 3, pp. 845-853, March 2019.

[46] M. Scherhäufl, M. Pichler, and A. Stelzer, "UHF RFID Localization Based on Evaluation of Backscattered Tag Signals", IEEE Transactions on Instrumentation and Measurement, vol. 64, no. 11, pp. 2889-2899, Nov. 2015.

[47] E. Di Giampaolo and F. Martinelli, "Multiple Baseline Synthetic Array for UHF RFID Localization", 2019 IEEE International Conference on RFID Technology \& Application (RFID-TA), Pisa, 2019, pp. 348-352.
[48] L. Qiu, Z. Huang, N. Wirström and T. Voigt, "3DinSAR: Object 3D localization for indoor RFID applications," 2016 IEEE International Conference on RFID (RFID), Orlando, FL, 2016, pp. 1-8.

[49] A. Tzitzis et al., "Localization of RFID Tags by a Moving Robot, via Phase Unwrapping and Non-Linear Optimization," IEEE Journal of Radio Frequency Identification, vol. 3, no. 4, pp. 216-226, Dec. 2019.

[50] A. Tzitzis, et al., "3D Localization of RFID Tags with a Single Antenna by a Moving Robot and Phase ReLock," 2019 IEEE International Conference on RFID Technology and Applications (RFID-TA), Pisa, Italy, 2019, pp. 273-278.

[51] A. Motroni, F. Bernardini, P. Nepa, P. Tripicchio, and M. Unetti, "Towards a Multi-antenna approach for UHF-RFID tag 3D localization with a Synthetic Aperture Radar Method," 2019 4th International Conference on Smart and Sustainable Technologies (SpliTech), Split, Croatia, 2019, pp. 1-7.

[52] F. Bernardini et al., "Particle Swarm Optimization in SAR-based Method enabling Real-Time 3D Positioning of UHF-RFID Tags," IEEE Journal of Radio Frequency Identification, doi: 10.1109/JRFID.2020.3005351.

[53] Nonuniform Sampling, Theory and Practice (ed. F. Marvasti), Kluwer Academic/Plenum Publishers, New York, 2000.

[54] Impinj, Low Level User Data Support, 2013. [Online]. Available: https://support.impinj.com/hc/en-us/articles/202755318-ApplicationNote-Low-Level-User-Data-Support

[55] G. Fornaro, F. Lombardini, and F. Serafino, "Three-dimensional multipass SAR focusing: Experiments with long-term spaceborne data," IEEE Transactions on Geoscience and Remote Sensing, vol. 43, no. 4, pp. 702-714, Apr. 2005.

[56] Optitrack, 2018. [Online]. Available: https://optitrack.com/motioncapture-robotics/.

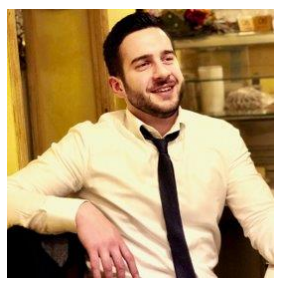

Fabio Bernardini (Student Member, IEEE) received the B.E. and M.E. degrees in telecommunications engineering from the University of Pisa, Pisa, Italy, in 2016 and 2018, respectively, where he is currently pursuing the Ph.D. degree in information engineering. His current research interests include indoor radio localization systems (with a specific focus on UHF-RFID phasebased algorithms), passive RFID sensing and RFID applications for the internet of things and Industry 4.0. He is IEEE member from February 2019 and he is President of the IEEE student branch of Pisa from February 2020.

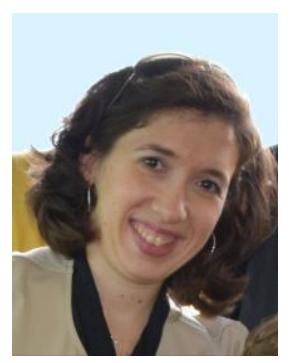

Alice Buffi (Member, IEEE) received the B.S. and M.S. (summa cum laude) degrees in Telecommunications Engineering and the Ph.D. degree in Applied Electromagnetism in Electrical and Biomedical Engineering, Electronics, Smart Sensors, Nanotechnologies with Doctor Europaeus label from the University of Pisa, Italy, in 2006, 2008 and 2012, respectively. She was a Visiting Ph.D. Student with the Queen Mary University of London, London, U.K., in 2012. Since 2012 she has been with the University of Pisa, where she is currently an Assistant Professor with the Department of Energy, Systems, Territory and Construction Engineering. She co-authored several international journal papers and international conferences contributions, one European patent and one Italian patent application. Her current research topics include measurement methods for localization and classification with UHF-RFID systems. She is an Associate Editor of the IEEE JOURNAL OF RADIO FREQUENCY IDENTIFICATION. Dr. Buffi was a recipient of the Young Scientist Award from the International Union of Radio Science, Commission B, in 2013 and 2016. She was also a recipient of the Best Paper Award at the IEEE 2019 RFID-TA Conference. 


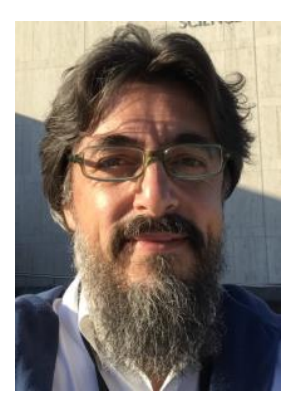

Daniele Fontanelli (Senior Member, IEEE) received the M.S. degree in Information Engineering in 2001, and the Ph.D. degree in Automation, Robotics and Bioengineering in 2006, both from the University of Pisa, Pisa, Italy. He was a Visiting Scientist with the Vision Lab of the University of California at Los Angeles, Los Angeles, US, from 2006 to 2007. From 2007 to 2008, he has been an Associate Researcher with the Interdepartmental Research Center "E. Piaggio", University of Pisa. From 2008 to 2013 he joined as an Associate Researcher the Department of Information Engineering and Computer Science and from 2014 the Department of Industrial Engineering, both at the University of Trento, Trento, Italy, where he is now an Associate Professor. He has authored and co-authored more than 150 scientific papers in peer-reviewed top journals and conference proceedings. He is currently an Associate Editor for THE IEEE TRANSACTIONS ON INSTRUMENTATION AND MEASUREMENT and for the IET SCIENCE, MEASUREMENT \& TECHNOLOGy Journal. From 2018 he is also an Associate Technical Program Committee Member for the IEEE/RSJ International Conference on Intelligent Robots and Systems. His research interests include distributed estimation and control, human localization algorithms, synchrophasor estimation, clock synchronization algorithms, realtime estimation and control, wheeled mobile robots estimation and control, and service robotics.

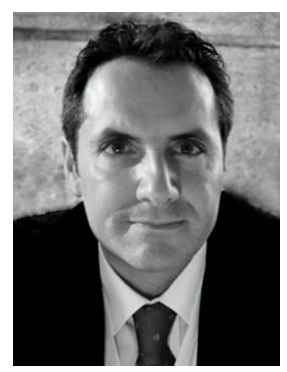

David Macii (Senior Member, IEEE) received the M.S. degree in Electronics Engineering and the $\mathrm{Ph} . \mathrm{D}$. degree in Information Engineering from the University of Perugia, Perugia, Italy, in 2000 and in 2003 , respectively. He was a visiting researcher at the University of Westminster, London, UK in 2002, at the Advanced Learning and Research Institute of the University of Lugano, Lugano, Switzerland, between 2004 and 2005 and a Fulbright Research Scholar at the Berkeley Wireless Research Center, University of California at Berkeley, Berkeley, USA between 2009 and 2010. Currently, he is an Associate Professor with the Department of Industrial Engineering University of Trento, Trento, Italy. He is author and co-author of about 140 papers published in books, scientific journals, and international conference proceedings. Since 2018 he has been an Editor for the journal MEASUREMENT. His research interests include measurement and estimation techniques based on digital signal processing for a variety of applications.

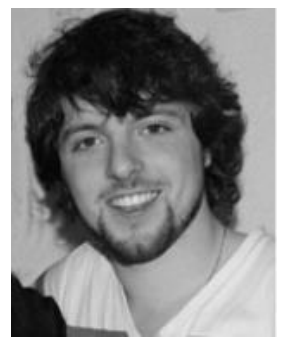

Valerio Magnago received the M.S. degree in mechatronics engineering from the University of Trento, Trento, Italy, in 2016, where he is currently pursuing the Ph.D. degree in information and communication technologies with the Department of Information Engineering and Computer Science. In 2019, he was a Visiting Ph.D. Student with the Technical University of Munich (TUM), Munich, Germany. He is also a member of the Embedded Electronics and Computing Systems Group, University of Trento. His main research areas are robotics, vision systems, and indoor positioning techniques.

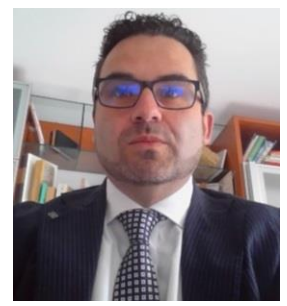

Mirko Marracci (Member, IEEE) was born in Viareggio, Italy, in 1977. He received the M.S. degree in electrical engineering and the Ph.D. degree in energetic from the University of Pisa, Pisa, Italy, in 2001 and 2005, respectively. Since 2005, he has been with the Department of Electrical Systems and Automation (now DESTeC), University of Pisa, where he is currently an Associate Professor of Electrical Measurements. His current research interests include characterization of materials, magnetic hysteresis, electromagnetic compatibility, magnetic shielding, and sensors.

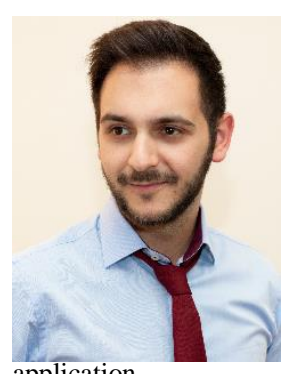

Andrea Motroni (Student Member, IEEE) received the B.E. and M.E. (summa cum laude) in Telecommunications Engineering from the University of Pisa, Pisa, Italy, in 2015 and 2017, respectively. Since 2017 he is attending the Ph.D. course in Information Engineering at the University of Pisa. His current research interests include indoor radio localization systems (with specific focus on UHF RFID phase-based algorithms), passive RFID sensing and RFID applications for Internet of Things and Industry 4.0. He co-authored one Italian patent

application.

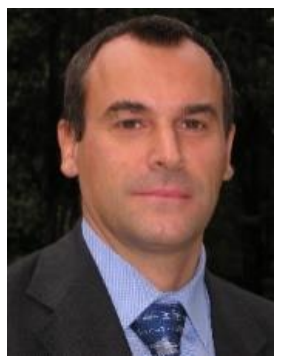

Paolo Nepa (Senior Member, IEEE) received the Laurea degree (summa cum laude) in electronics engineering from the University of Pisa, Pisa, Italy, in 1990. Since 1990, he has been with the Department of Information Engineering, University of Pisa, where he is currently a Full Professor. In 1998, he was a Visiting Scholar at the Electro Science Laboratory (ESL), The Ohio State University (OSU), Columbus, OH, USA, where he was involved in efficient hybrid techniques for the analysis of large antenna arrays. His current research interests include the design of wideband and multiband antennas, and antennas optimized for near-field coupling and focusing. In the context of UHF-RFID systems, he is working on techniques for radiolocalization of either tagged objects or readers. He has co-authored more than 300 international journal articles and conference contributions. Dr. Nepa is a member of the Technical Advisory Board of URSI Commission B - Fields and Waves. He served as the General Chair of the IEEE RFID-TA 2019 International Conference. Since 2016, he has been serving as an Associate Editor for the IEEE ANTENNAS AND WIRELESS PROPAGATION LETTERS. He was a recipient of the Young Scientist Award from the International Union of Radio Science, Commission B, in 1998.

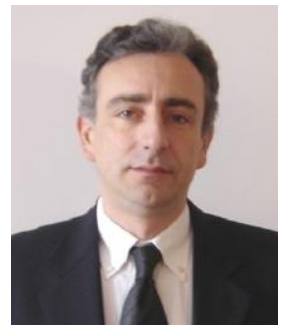

Bernardo Tellini (Senior Member, IEEE) was born in Pisa, Italy, in 1969. He received the M.S. (Laurea) and Ph.D. degrees in electrical engineering from the University of Pisa, Pisa, in 1993 and 1999, respectively. Since 2000 , he has been with the University of Pisa, where he is currently a Full Professor of electrical measurements with the Department of Energy, Systems, Territory and Construction Engineering. His current research interests include characterization of magnetic materials, electromagnetic emission associated with fast electromagnetic transients, sensors, and transducers for pulsed power applications, and magnetohydrodynamics problems. Dr. Tellini is a member of the IEEE Instrumentation and Measurement Society and the IEEE Magnetics Society. He is also the Chair of the IEEE Italy Section. He was the Chair of European Pulsed Power Laboratories from2010 to 2014 and the General Chair of the International Instrumentation and Measurement Technology Conference in 2015 . 